\title{
A Mechanism of Spark Motion in Inner Acceleration Region to Investigate Subpulse Drifting in Pulsars
}

\author{
Rahul Basu ${ }^{1,2}$, Dipanjan Mitra ${ }^{2,3}$, George I. Melikidze 2,4 \\ 1 Inter-University Centre for Astronomy and Astrophysics, Pune, 411007, India; rahulbasu.astro@gmail.com \\ 2 Janusz Gil Institute of Astronomy, University of Zielona Góra, ul. Szafrana 2, 65-516 Zielona Góra, Poland \\ 3 National Centre for Radio Astrophysics, Tata Institute of Fundamental Research, Pune 411007, India \\ 4 Evgeni Kharadze Georgian National Astrophysical Observatory, 0301, Abastumani, Georgia
}

3 June 2020

\begin{abstract}
Coherent radio emission in pulsars is excited due to instabilities in a relativistically streaming non-stationary plasma flow, which is generated from sparking discharges in the inner acceleration region (IAR) near the stellar surface. A number of detailed works have shown the IAR to be a partially screened gap (PSG) dominated by non-dipolar magnetic fields with continuous outflow of ions from the surface. The phenomenon of subpulse drifting is expected to originate due to variable $\mathbf{E} \times \mathbf{B}$ drift of the sparks in PSG, where the sparks lag behind corotation velocity of the pulsar. Detailed observations show a wide variety of subpulse drifting behaviour where subpulses in different components of the profile have different phase trajectories. But the drifting periodicity is seen to be constant, within measurement errors, across all components of the profile. Using the concept of sparks lagging behind corotation speed in PSG as well as the different orientations of the surface non-dipolar magnetic fields we have simulated the expected single pulse behaviour in a representative sample of pulsars. Our results show that the different types of drifting phase behaviour can be reproduced using these simple assumptions of spark dynamics in a non-dipolar IAR.
\end{abstract}

Key words: pulsars: general

\section{INTRODUCTION}

One of the most intriguing features seen in the radio emission from pulsars involve the phenomenon of subpulse drifting, where systematic periodic shifts in individual components of a single pulse, known as subpulses, are seen within the pulse window (Drake \& Craft 1968). A complete physical understanding of this phenomenon is still absent, however the most successful explanation is provided in the work of Ruderman \& Sutherland (1975, hereafter RS75). According to the RS75 model an inner acceleration region (IAR) exists above the pulsar polar cap, where sparking discharges generate a spark associated non-stationary plasma flow, which stream relativistically along the open magnetic field lines and leave the pulsar magnetosphere as relativistic pulsar wind (Goldreich \& Julian 1969). The non-stationary flow is necessary to excite the coherent radio emission (Asseo \& Melikidze 1998; Melikidze et al. 2000; Mitra et al. 2009; Melikidze et al. 2014; Lakoba et al. 2018), while the drift motion of the spark associated plasma column results in subpulse drift. In the RS75 model the prototype for the IAR is the inner vacuum gap (IVG), which is formed in pulsars with $\boldsymbol{\Omega} \cdot \mathbf{B}<0$ above the polar cap, where $\boldsymbol{\Omega}(=2 \pi / P)$ is the an- gular velocity of pulsar with period $P$, and $\mathbf{B}$ is the magnetic field. It was postulated that due to the high binding energy of ions the positive charges cannot escape above the polar cap to screen the electric field and hence the IVG forms. The potential drop in the IVG is extremely high, about $10^{12} \mathrm{~V}$, and several isolated discharges mediated via magnetic $e^{-} e^{+}$ pair creation is setup in the gap. The electric field in the gap separates the pairs and accelerate the charges in opposite directions. The $e^{-}$travel downwards towards the stellar surface and the $e^{+}$upwards, producing high energy photons via curvature radiation and/or inverse compton scattering, which lead to a pair cascade. This process continues till the entire potential drop along the IVG is screened, i.e., when the charge density reaches the so called GoldreichJulian density $\left(\rho_{G J}\right)$. During this interval the discharge also grows in the perpendicular direction into adjacent field lines and eventually a full formed spark develops. Once the IVG is screened, the spark associated plasma column leaves the gap and the electric potential appears once again for the sparking process to commence. Several studies have shown that the efficiency of the pair cascade process requires the presence of strong surface non-dipolar magnetic fields (e.g. 
Timokhin \& Harding 2019) with radius of curvature $\left(\rho_{c}\right)$ of about $10^{5}-10^{6} \mathrm{~cm}$. In contrast $\rho_{c}$ for dipolar magnetic field is around $10^{8} \mathrm{~cm}$, and hence the presence of highly nondipolar surface magnetic field in the IAR is essential ${ }^{1}$. When the charge density in sparks reaches $\rho_{G J}$, the force-free condition is achieved and the plasma column corotates with the star due to $\mathbf{E} \times \mathbf{B}$ drift. However, during the sparking process the charge density in the gap is below $\rho_{G J}$, resulting in the charges lagging behind the corotation motion. This phenomenon is responsible for the observed subpulse drifting in observed radio emission (see e.g. RS75, Allen 1985; Szary 2013; Basu et al. 2016; Mitra et al. 2020).

Detailed classification studies of the observed drifting behaviour in the pulsar population have been carried out in the literature (Rankin 1986; Weltevrede et al. 2006, 2007; Basu et al. 2016; Basu \& Mitra 2018; Basu et al. 2019a). Subpulse drifting shows a wide variety of subpulse motion characterised by phase variations in fluctuation spectral analysis (Backer 1973; Backer et al. 1975). The drifting periodicity $\left(P_{3}\right)$, the interval at which the subpulses repeat at any location within the pulse window, is identical across all components in the pulsar profile despite large phase variations between them. In pulsars where a central core emission is seen surrounded by one or two conal pairs, subpulse drifting is absent in the central core component and only seen in the surrounding cones. The systematic drifting behaviour has been classified by Basu et al. (2019a) into three major categories:

a) Coherent phase-modulated drifting where the subpulses continuously shift from one edge of the pulse window to the other. The phase show large monotonic variations across the profile which are usually non-linear.

b) Switching phase-modulated drifting is seen in pulsars with more than one component in the profile where subpulses show systematic variations across each component resulting in large phase variations, but there are sudden shifts between adjacent components. In certain cases the subpulses show opposite sense of variation in different components with slopes of phase variations showing opposite signs. This phenomenon is known as bi-drifting (Champion et al. 2005; Weltevrede 2016; Basu \& Mitra 2018; Basu et al. 2019b).

c) Low-mixed phase-modulated drifting is also seen in pulsars with multi-component profiles where the subpulses do not show large shifts during drifting. The resulting phase variations are relatively flat across each component.

One of the primary challenges is to understand these wide variety seen in the drifting behaviour from the perspective of the sparking model, which forms the main focus of this work. In section 2 we discuss the origin of subpulse drifting in the physically consistent Partially Screened Gap (PSG, Gil et al. 2003) model of the IAR. Section 3 presents a simplified mechanism to generate single pulses exhibiting subpulse drifting, which is subsequently used is section 4 to study the drifting behaviour in different surface magnetic field config-

1 There are also indications of the presence of non-dipolar magnetic fields near the surface from X-ray observations of normal period pulsars (see Arumugasamy \& Mitra 2019; Geppert 2017; Sznajder \& Geppert 2020). urations. A short discussion regarding the implications of these simulations on subpulse drifting as well as their limitations are presented in section 5 .

\section{SUBPULSE DRIFTING IN THE PARTIALLY SCREENED GAP MODEL}

\subsection{Partially Screened Gap Model}

Formation of the IVG in the RS75 model requires binding energy at the stellar surface to be sufficiently high to prevent positive ions to escape. A number of subsequent studies have found the above assumptions to be inadequate, particularly when the polar cap surface is constantly bombarded by backstreaming electrons during the sparking process (Cheng \& Ruderman 1980; Gil et al. 2003). This can cause the polar cap temperatures to rise above $10^{6} \mathrm{~K}$, which is sufficient for a continuous outflow of positively charged ions from the stellar surface. Gil et al. (2003) suggested that the IAR is in fact a partially screened gap (PSG), with screening factor $\eta=1-\rho_{i} / \rho_{G J}$, where $\rho_{i}$ is the charge density due to production of ions in the IAR. The PSG is thermally regulated around the critical temperature $\left(T_{i}\right)$ of ion free flow from the surface, where any drop in temperature below this critical value is accompanied by sparking discharges to quickly reheat the surface back to the critical level. One of the most important features of the PSG is providing a mechanism for the sparking regions to be stable within the IAR. The region between sparks in the PSG is screened due to the presence of plasma with charge density $\rho_{G J}$, and hence no additional particle acceleration can take place. On the other hand in the IVG the region between the sparks is vacuum, where unscreened electric field exist and hence can discharge due to pair creation. As a result the sparking discharges cannot be confined at any location on the surface, but moves continually opposite to the principal normal of the curvature of the local magnetic field lines (Cheng \& Ruderman 1977, 1980). In contrast in the PSG the development of pair cascade is restricted to localised sparking regions where the surface temperature is below the critical level and is inhibited in the region between the sparks.

The behaviour of subpulse drifting further justifies the requirement of PSG in IAR. Basu et al. (2016) found the measured drifting periodicity to be anti-correlated with the spin-down energy loss $(\dot{E})$, with an estimated dependence of $P_{3} / P \propto \dot{E}^{-0.6 \pm 0.1}$. The PSG nature of the IAR currently provides the only explanation for this observed dependence. The IAR potential is screened by the screening factor which gives an estimate of the speed of the sparks in IAR, and consequently the periodicity under certain approximations can be estimated as $P_{3} / P=1 / 2 \pi \eta\left|\cos \alpha_{l}\right|$, where $\alpha_{l}$ is the angle the local non-dipolar magnetic field makes with the rotation axis (Szary 2013; Mitra et al. 2020). It can be shown that in typical pulsars $\eta \sim 0.1$, and is proportional to the spin-down energy loss as $\eta \propto \sqrt{\dot{E}}$, which gives the observed dependence (see discussions in Basu et al. 2016; Mitra et al. 2020).

In figure 1, the time evolution of the sparking process in the PSG model is shown. When the surface temperature $\left(T_{S}\right)$ in the polar cap exceeds $T_{i}$, the ions steadily flow out from the stellar surface, populating the IAR with $\rho_{G J}$ which 
$\vec{E} \cdot \vec{B}=0$

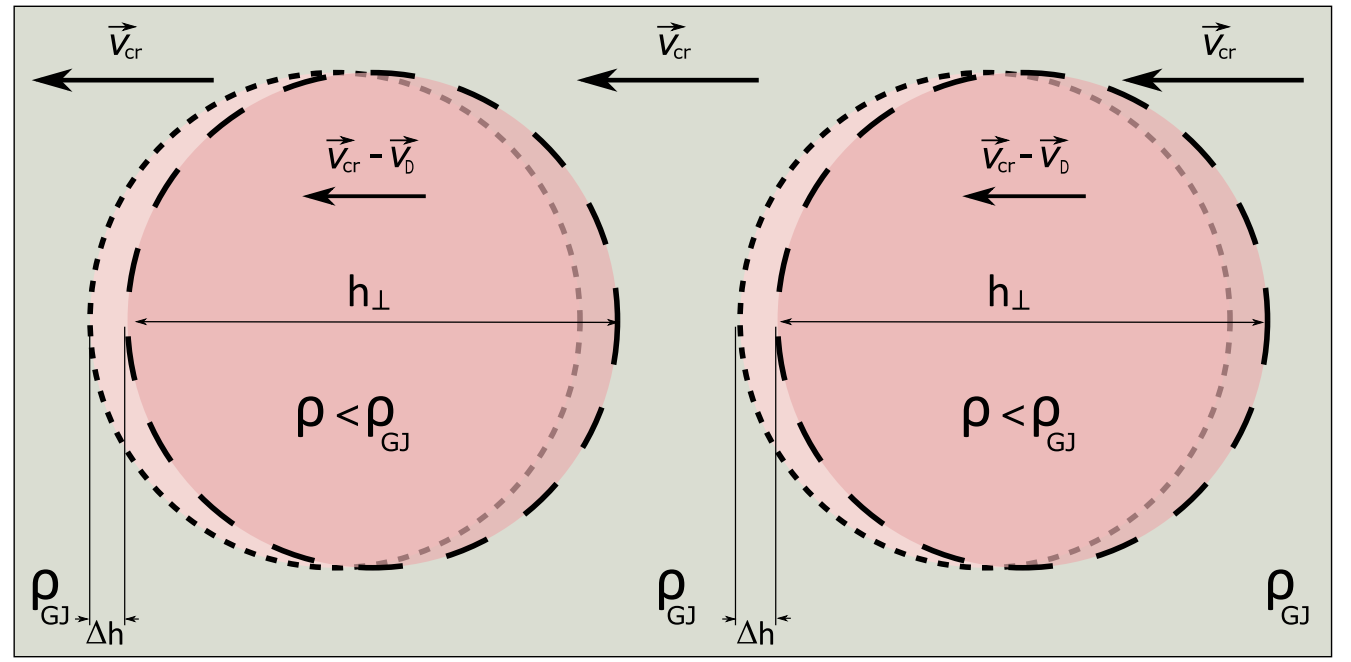

Figure 1. The figure shows a top-down view of the time evolution of the sparking process from the observer's frame, as proposed in the PSG model. The rectangular box corresponds to the polar cap with $\vec{\Omega} \cdot \vec{B}<0$, where two distinct sparking regions (circles with lighter shade to darker shade of pink) with typical size $h_{\perp}$ are shown. The direction of rotation of the pulsar is from right to left indicated by the corotation velocity, $\overrightarrow{v_{c r}}$. The region outside the polar cap has $\vec{E} \cdot \vec{B}=0$ and also corotates with the pulsar. The pale green region corresponds to the region between sparks filled with charge density $\rho_{G J}$. In the sparking region the charge density is $\rho<\rho_{G J}$. With the progression of time the initial spark (lighter shade circle) is replaced by a subsequent spark (shown in darker shade) which is shifted by a distance $\Delta h$ opposite to the corotation direction and moving with an effective velocity $\overrightarrow{v_{c r}}-\overrightarrow{v_{D}}$.

screens the electric potential along the gap. Sparking commences at localised regions where $T_{S}$ drops below critical level such that the charge density is reduced by a factor $\eta$ and large potential difference builds up along the gap. This instigates $e^{-} e^{+}$pair cascades in this sparking region, with relativistic back-streaming electrons bombarding the stellar surface and heating the region below the spark. The sparking process continues until $T_{s} \gtrsim T_{i}$ is reached, when the ions can freely flow out of the stellar surface and screen the electric potential along the gap with $\rho_{G J}$. In the absence of electric field, intense pair production terminates causing the sparking process to stop. The typical timescale over which this full spark develops is estimated to be $t_{s p} \sim 10 \mu \mathrm{s}$ (see e.g. Gil et al. 2003). The back-streaming electrons during $t_{s p}$ lag behind the corotation of the star and hence the peak heating region also lags behind along the corotation direction. The sparking discharges grow to attain lateral width $h_{\perp}$ whose estimates in the PSG model is given as (see eq.4 and discussion above it in Mitra et al. 2020)

$h_{\perp}=2.6 \frac{T_{6}^{2}}{\eta b \cos \alpha_{l}}\left(\frac{P}{\dot{P}_{-15}}\right)^{0.5} \mathrm{~m}$.

Here $T_{6}$ is the surface temperature in million $\mathrm{K}$ and $b=$ $B_{s} / B_{d}$ where $B_{s}$ is the surface non-dipolar magnetic field and $B_{d}$ the equivalent dipolar case. For a typical pulsar these parameters can be approximated as $\eta=0.1, b=10$, $T_{6}=P=\dot{P}=\left|\cos \alpha_{l}\right|=1$, and we have $h_{\perp} \sim 2.6 \mathrm{~m}$. The velocity of lagging behind process in a PSG can be estimated as $\eta v_{c r}$, where $v_{c r}$ is the corotation velocity. The maximum heated region on the surface is shifted from the center of the spark along the corotation direction by $\Delta h_{\perp}=\eta v_{c r} t_{s p}$. Using $\eta=0.1, v_{c r} \sim 10^{6}-10^{7} \mathrm{~cm} / \mathrm{s}$ (see section 5.1), and $t_{s p} \sim 10^{-5}$ $\mathrm{s}$, we have $\Delta h_{\perp} \sim 10 \mathrm{~cm}$. When the spark associated plasma column reaches $\rho_{G J}$, it corotates with the rest of the star till it leaves the gap region due to inertia. In this process the hot region that was formed below the spark lags slightly behind corotation by a distance $\Delta h_{\perp}$. The cooling timescale of this hot region is of the order of nanoseconds, while the gap emptying time of the plasma column is microseconds which is several times longer. Thus the cooling is most efficient in a region shifted by $\Delta h_{\perp}$ from the spark center, behind the corotation direction, where the condition $T_{S}<T_{i}$ is satisfied. After the plasma column empties a potential drop appears along the IAR causing initiation of the subsequent sparking discharge. The discharges grows both along the horizontal and vertical direction to form a new spark. The locus of this spark is shifted by $\Delta h_{\perp}$ behind the previous one.

As the plasma column from the spark escapes the IAR, they form a secondary cloud of pair plasma with high multiplicity $\left(\sim 10^{5}\right.$, Sturrock 1971; Timokhin \& Harding 2019) and typical length $c t_{s p} \sim 3 \mathrm{~km}$, along the magnetic field lines. The secondary plasma clouds move outwards along the open field lines and generate radio emission at heights of $\sim 500 \mathrm{~km}$ from the surface, where the magnetic field is dipolar (Mitra 2017). The average emission from several thousand ${ }^{2}$ such secondary plasma columns is seen as a subpulse in the pulse window. As the next spark is shifted behind the corotation direction by $\Delta h_{\perp}$, the subsequent secondary

${ }^{2}$ For $P=1 \mathrm{~s}$ and pulse window around $10 \%$ of the period, the subpulse usually covers between a fifth and a third of the window making it several tens of milliseconds in width. Hence, several thousand secondary plasma columns, with typical timescales of tens of microseconds, make up a subpulse. 
plasma clouds are also formed with equivalent shifts across the magnetic field. The physical shift between two consecutive clouds are very small, less than $5 \%$ of the lateral size. However, they represent a continuous process such that over time the location of the subpulse within the pulse window is clearly shifted which is seen as subpulse drifting.

We consider the IAR to be tightly packed with equidistant sparks (Gil \& Sendyk 2000), and subsequent sparks are formed at a location slightly behind the corotation direction. The maximum number of sparks $\left(n_{s p}\right)$ along any diametric cross section of the IAR under such conditions can be estimated using the PSG model as (Mitra et al. 2020)

$n_{s p} \simeq 15 \frac{\eta b^{0.5} \cos \alpha_{l}}{T_{6}^{2}}\left(\frac{\dot{P}_{-15}}{P^{2}}\right)^{0.5}$.

The parameters, $\eta, b, \alpha_{l}$ and $T_{6}$ are not well constrained, but if we consider typical values $\eta=0.1, b=10,\left|\cos \alpha_{l}\right|=T_{6}=1$, we have $n_{s p} \sim 5$ for a pulsar with $P=1 \mathrm{~s}$. The pulsar J21443933 , with $P=8.5 \mathrm{~s}$ and a single component in its profile, illustrates the viability of these estimates. If we assume the magnetic field configuration to be highly non-dipolar in this case with $b=40$, then $n_{s p} \sim 1$ implying only a single spark can be accommodated within the IAR. This concept was used by Mitra et al. (2020) to predict the death line in the $P-\dot{P}$ diagram and provides a tight constrain to the population distribution (see figure 6 in Mitra et al. 2020).

\subsection{Lagging behind co-rotation in Inner Acceleration Region}

The phenomenon of subpulse drifting arising due to sparks lagging behind corotation for any arbitrary geometry, has been discussed in earlier studies for simpler magnetic field configurations, where surface fields are dominated by the radial component (Allen 1985; Szary 2013; Basu et al. 2016; Mitra et al. 2020). To further elucidate this concept we explore the electrodynamics for a general magnetic field structure. Let us consider two reference frames primed and unprimed, where the primed frame corresponds to the corotating neutron star and the unprimed frame represent the observer. The relation between the electric fields in the two frames, near the polar cap of a slowly rotating pulsar $(\Omega R / c \ll 1)$, can be expressed as :

$\mathbf{E}^{\prime}=\mathbf{E}+\frac{1}{c} \mathbf{v} \times \mathbf{B}$

where $\mathbf{v}=\boldsymbol{\Omega} \times \mathbf{r}$, is the rotation velocity of the puslar.

A simple schematic to explain the relation between the fields and velocities in a moving frame and rest frame is shown in figure 2. In this figure a test charge particle is considered to be at rest in the $\mathrm{x}-\mathrm{z}$ plane, and the magnetic field $\mathbf{B}=\left(B_{x}, 0, B_{z}\right)$ is confined in the $\mathrm{x}-\mathrm{z}$ plane. Now if we consider an observer moving along the negative $\mathrm{x}$-axis with velocity $\mathbf{v}_{o}=-v_{o} \hat{x}$, the test particle will appear to move in the opposite direction in the observer's frame. An electric field $\mathbf{E}_{o}$ appears in this frame along the y-axis given by,

$$
\begin{aligned}
\mathbf{E}_{o} & =-\frac{1}{c} \mathbf{v}_{o} \times \mathbf{B} \\
& =\frac{1}{c} v_{o} B_{z} / c \hat{y}
\end{aligned}
$$

In the observer's frame the velocity of the charged particle

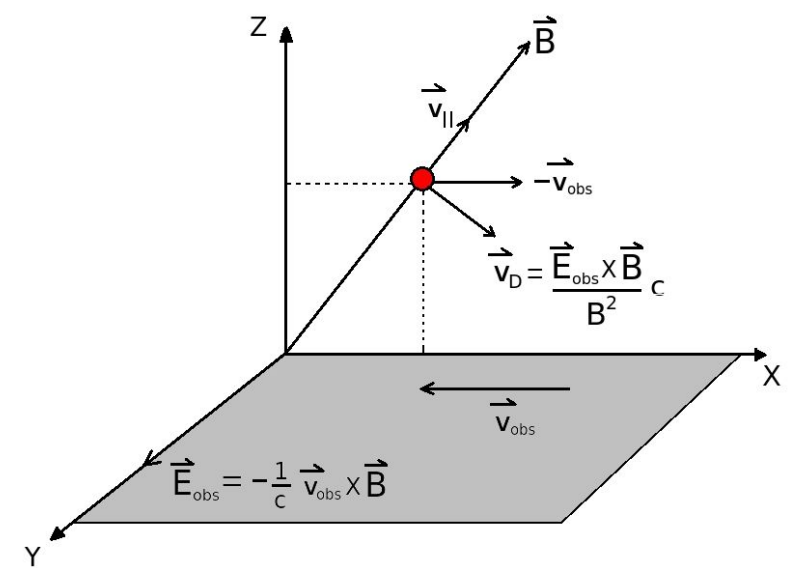

Figure 2. The figure presents a simplified schematic of electromagnetic field configurations and velocities for a charge particle at rest in a magnetic field as seen from the perspective of a moving observer. The cartesian coordinate system is setup in rest frame of the particle which also contains magnetic field $\mathbf{B}$. The particle and $\mathbf{B}$ are setup in the $\mathrm{x}-\mathrm{z}$ plane for simplicity of visualization. There is no electric field in this frame. An observer moving with $\mathbf{v}_{o b s}$ along the $\mathbf{x}$-axis sees the particle move along $-\mathbf{v}_{o b s}$, with $\mathbf{v}_{D}$ and $\mathbf{v}_{\|}$lying in the $\mathrm{x}-\mathrm{z}$ plane. The electric field $\mathbf{E}_{\text {obs }}$ also appears along $\mathrm{y}$-axis to the observer due to frame transformation.

can be expressed in terms of two perpendicular components, the drift velocity $\mathbf{v}_{D}$ perpendicular to the magnetic field and $\mathbf{v}_{\|}$along magnetic field, such that $-\mathbf{v}_{o}=\mathbf{v}_{D}+\mathbf{v}_{\|}$. The $\mathbf{E} \times \mathbf{B}$ drift velocity of particle $\mathbf{v}_{D}$ is given as :

$$
\begin{aligned}
\mathbf{v}_{D} & =c \frac{\mathbf{E}_{o} \times \mathbf{B}}{B^{2}} \\
& =\frac{v_{o}}{B^{2}}\left(-B_{z}^{2} \hat{x}+B_{x} B_{z} \hat{z}\right) .
\end{aligned}
$$

An analogous behaviour is seen in pulsars. When full corotational charge density $\rho_{G J}$ exist in the pulsar magnetosphere the corotational electric field $\mathbf{E}^{\prime}=0$ in the rest frame of the pulsar. It follows from eq. (3) that in the observer's frame the corotation electric field is $\mathbf{E}_{\mathbf{c}}=-\frac{1}{c}(\boldsymbol{\Omega} \times \mathbf{r}) \times \mathbf{B}$. We choose a coordinate system centered on the neutron star, and consider a slowly rotating pulsar with angular velocity $\boldsymbol{\Omega}$ aligned along the z-axis, such that $\boldsymbol{\Omega}=\Omega \hat{z}$, where $\hat{z}=$ $\cos \theta \hat{r}-\sin \theta \hat{\theta}$, and magnetic field given by $\mathbf{B}=\left(B_{r}, B_{\theta}, B_{\phi}\right)$. The corotation electric field $\mathbf{E}_{c}=\left(E_{r}^{c}, E_{\theta}^{c}, E_{\phi}^{c}\right)$ in the above coordinate system at a point $\mathbf{r}=(r, \theta, \phi)$ is given as :

$$
\begin{aligned}
& E_{r}^{c}=\frac{\Omega r \sin \theta B_{\theta}}{c}, \\
& E_{\theta}^{c}=-\frac{\Omega r \sin \theta B_{r}}{c}, \\
& E_{\phi}^{c}=0 .
\end{aligned}
$$

The electric field in eq.(6) is a more generalised version of eq. (4) and is perpendicular to the magnetic field. Thus the drift velocity $\left(\mathbf{v}_{D}^{c}\right)$, which is the component of the corotation velocity $(\boldsymbol{\Omega} \times \mathbf{r})$ perpendicular to the local magnetic field, is given as $\mathbf{v}_{D}^{c}=c(\mathbf{E} \times \mathbf{B}) / B^{2}$. Using eq.(6) we obtain $\mathbf{v}_{D}^{c}=$ 
$\left(v_{D, r}^{c}, v_{D, \theta}^{c}, v_{D, \phi}^{c}\right)$ to be :

$v_{D, r}^{c}=-\frac{\Omega r B_{r} B_{\phi} \sin \theta}{B^{2}}$,

$v_{D, \theta}^{c}=-\frac{\Omega r B_{\theta} B_{\phi} \sin \theta}{B^{2}}$,

$v_{D, \phi}^{c}=\frac{\Omega r\left(B_{r}^{2}+B_{\theta}^{2}\right) \sin \theta}{B^{2}}$,

which is a more generalised form of eq.(5).

The steady state condition where the magnetosphere is filled with corotational charge density $\rho_{G J}$, breaks down in the IAR where a continuous outflow of plasma is setup, leaving behind a gap. As the charge density empties in the gap region an electric field $\mathbf{E}_{\|}$along the magnetic field appears in the pulsar frame in addition to perpendicular component of the electric field $-\mathbf{E}_{c}$ which is opposite to the corotation field derived above. It is often reported in the literature that the charge particles in the IAR would see an electric field such that they rotate within the IAR around the magnetic axis (Ruderman \& Sutherland 1975; van Leeuwen \& Timokhin 2012; Szary \& van Leeuwen 2017). However, in the absence of any external electric field, a charge in IAR would lag behind corotation in the observer's frame, and rotate opposite to the corotation direction in the pulsar frame due to the $-\mathbf{E}_{c}$ field. It is clear from eq. (7) that the $v_{D, \phi}^{c}$ has the same sign throughout the polar $\mathrm{cap}^{3}$ and hence the charges cannot rotate around the magnetic axis.

There are two primary features of the plasma generation process in the IAR discussed above. Firstly, the sparking is associated with a continuous lagging behind corotation motion, where every subsequent spark is formed slightly behind the corotation direction. Secondly, the surface of the neutron star is dominated by non-dipolar magnetic field while the magnetic field in emission region higher up in the magnetosphere is purely dipolar in nature. As a consequence, the sparks lagging behind in the IAR will have different paths in the emission region. Using these basic assumptions we demonstrate in this work that the majority of observed subpulse drifting behaviour in pulsars, outlined earlier, can be reproduced by considering different manifestations of surface non-dipolar fields.

\section{MODELLING SINGLE PULSE SEQUENCE}

In order to generate a single pulse sequence there are two primary inputs that are required, firstly, the nature of the magnetic field configuration, and, secondly, the nature of spark motion in IAR. We describe below our simplified model for the magnetic field configuration as well as the spark motion.

\subsection{Magnetic Field Structure}

We have used a simple configuration consisting of a star centered dipole (d) along with one or more surface dipoles $\left(\mathbf{m}_{i}\right.$, with $i=1,2, \ldots, N)$, anchored on the crust near the IAR, as our model for the non-dipolar magnetic field (Gil et al. 2002). In figure 3 a schematic of a configuration consisting

3 except in rare cases when magnetic inclination angle and hence $\theta$ is close to $0^{\circ}$ or $180^{\circ}$ of star centered dipole and one surface dipole is shown. The simulations are carried out in the rest frame of the pulsar which implies that observer's line of sight evolves with time and periodically cuts across the emission region, while the magnetic configuration remains fixed during the temporal evolution of the pulse sequence. The coordinate system is oriented such that the global dipole is located at the origin and along the $\mathrm{x}-\mathrm{z}$ plane, $\mathbf{d}=\left(d, \theta_{d}, 0^{\circ}\right)$. The surface field on the other hand is more complicated and requires two sets of variables for each dipole, one specifying the location $\mathbf{r}_{i}$ $=\left(r_{s}^{i}, \theta_{s}^{i}, \phi_{s}^{i}\right)$ and the other the magnetic field orientation $\mathbf{m}_{i}=\left(m^{i}, \theta_{m}^{i}, \phi_{m}^{i}\right)$. The detailed calculations of the resultant magnetic fields, in spherical coordinates, for the general configuration is shown in appendix A. For these simulations we have used a complete three dimensional solution of the magnetic line of force. This requires numerical solution of the system of differential equations in spherical coordinates,

$$
\begin{aligned}
\frac{\mathrm{d} \theta}{\mathrm{d} r} & =\frac{B_{\theta}^{d}+\sum_{i=1}^{N} B_{\theta}^{i}}{r\left(B_{r}^{d}+\sum_{i=1}^{N} B_{r}^{i}\right)} \\
\frac{\mathrm{d} \phi}{\mathrm{d} r} & =\frac{B_{\phi}^{d}+\sum_{i=1}^{N} B_{\phi}^{i}}{r\left(B_{r}^{d}+\sum_{i=1}^{N} B_{r}^{i}\right) \sin \theta}
\end{aligned}
$$

In accordance with Gil et al. (2002) we used typical parameters $m^{i}=0.001-0.05 d$ and $r_{s}^{i}=0.95 R_{S}$, where $R_{S}=10$ $\mathrm{km}$, the radius of neutron star. For the above parameters the surface dipole contributions are $\approx 0$ at heights of $50 R_{S}$, which was used as the initial condition to trace the field lines to the stellar surface. We have varied the remaining parameters, i.e, the location of the crust dipoles specified by $\theta_{s}^{i}, \phi_{s}^{i}$, as well as the dipole orientations using $\theta_{m}^{i}, \phi_{m}^{i}$, to generate different configurations of the surface field as required.

The pulsar magnetosphere is separated into the open and closed field line regions which are bound by the light cylinder radius $R_{L C}(=c P / 2 \pi)$. The radio emission originates at heights of $R_{E} \sim 50 R_{S}$ The pulsar magnetosphere is separated into the open and closed field line regions which are bound by the light cylinder radius $R_{L C}(=c P / 2 \pi)$. The radio emission originates at heights of $R_{E} \sim 50 R_{S}$ (Kijak \& Gil 1997; Mitra \& Rankin 2002; Kijak \& Gil 2003; Mitra \& Li 2004; Krzeszowski et al. 2009; Mitra 2017) along the open field lines. The opening angle of the open field line region is estimated from equation of dipolar fields as $\theta_{o}^{E}=\sin ^{-1}\left(\sqrt{R_{E} / R_{L C}}\right)$. The entire open field line region at the emission height is also referred to as emission beam. Using the boundary value of $\theta=\theta_{o}^{E}$ at $r=R_{E}$, and varying $\phi$, the boundary of the non-dipolar polar cap is estimated from the solutions of the field line equations (eq. 8). In figure 4 the polar cap boundaries for two different configuration of the non-dipolar fields are shown in addition to the purely dipolar polar cap. In each case the surface dipole is located $5^{\circ}$ offset from the center of the dipolar axis, the first case along $\theta$-axis and second along the $\phi$-axis. The figure shows that the polar cap is elongated and smaller in area compared to the dipolar polar cap which is circularly symmetric.

The pulsed emission is seen when the line of sight (LOS) traverses the open field lines in the emission region during 


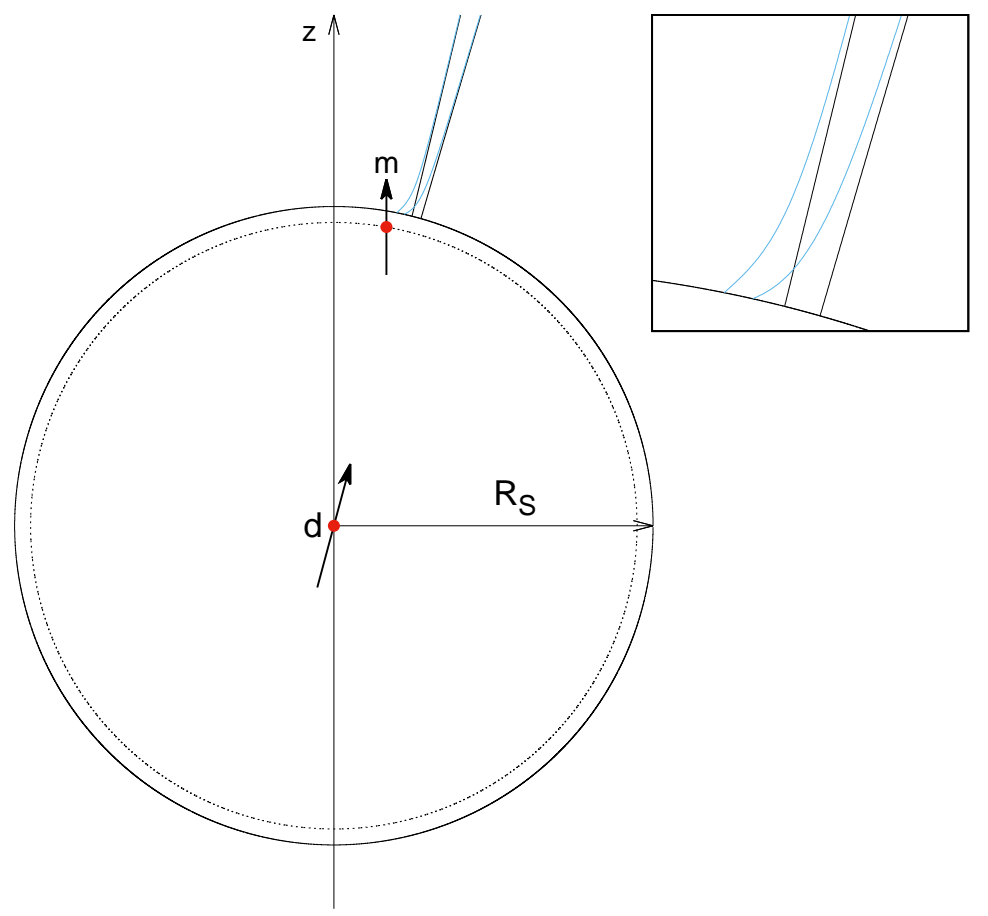

Figure 3. The figure shows a representation of the magnetic configuration comprising of a star centered dipole $\mathbf{d}=\left(d, 15^{\circ}, 0^{\circ}\right)$ inclined from the rotation axis, and a second dipole $\mathbf{m}_{s}=\left(0.001 d, 0^{\circ}, 0^{\circ}\right)$ anchored in the crust at $\mathbf{r}_{s}=\left(0.95 R_{S}, 10^{\circ}, 0^{\circ}\right)$. The resultant non-dipolar polar cap (the inset figure) is shifted from the dipolar polar cap but at heights of few times the stellar radius the dipolar field dominates.

the pulsar rotation. The LOS is characterized by the angle $\beta$ which corresponds to the angular separation between the LOS and the axis of the star centered dipole during their closest approach (Gil et al. 1984). Consequently, the profile shape and the observed subpulse motion is determined by the LOS traverse on the emission region as well as its corresponding track on the IAR. The track of the LOS on the IAR can be estimated by varying the $\phi$ coordinate across the open field line in the emission region, $r=R_{E}$, for a constant $\theta=\theta_{d}+\beta$, and for each field line estimating the equivalent location in the IAR. In figure 4 we also show the LOS in the IAR for the two non-dipolar configurations where $\beta=0^{\circ}$, i.e., the LOS cuts the emission beam centrally. As seen in the figure the LOS on the IAR are not centrally located due to the asymmetry of the non-dipolar fields.

\subsection{Spark Motion in Inner Acceleration Region}

The next step in generating single pulse sequence is establishing a dynamical sparking system in the IAR and associating the sparking process with subpulse variation. The sparks lag behind the star in the corotation direction, and move around the rotation axis with a drifting periodicity longer than the pulsar period. There are no studies explaining the detailed evolution of sparking process in IAR, which is also beyond the scope of this work. However, under the assumption that the IAR is tightly packed with sparks (Gil \& Sendyk 2000), the PSG model suggests a typical pulsar can accommodate a maximum of 5 sparks across any diametric cross section (see eq. 2 and discussion below it).
Beyond this basic understanding there is no detailed modelling which connects the spark width with the lateral size of the secondary plasma clouds above the IAR, as well as their relation to the subpulse width in the emission region. In a detailed work involving a large set of pulsars studied in the MSPES survey (Mitra et al. 2016), Skrzypczak et al. (2018) showed that a lower boundary in the distribution of component widths is present in pulsars, with a $P^{-0.5}$ dependence. The opening angle of the dipolar beam in the radio emission region also scales as $P^{-0.5}$, which suggests the presence of an upper limit for the number of components within the pulse window as well. Additionally, detailed studies of the emission beam suggests that a maximum of five components can be accommodated within the emission beam (Rankin 1993; Mitra \& Deshpande 1999) which is consistent with the estimations of the PSG model. In this work we have assumed the ratio of the diameter of the spark $(D)$ and polar cap radius $\left(r_{p}\right)$ is $D / r_{p} \sim 0.2$, for a maximum of 5 sparks present across the diameter of the polar cap. We have also assumed a direct one is to one correspondence between the sparks in the IAR and the subpulses in the emission region. This is obviously a simplistic approximation for reproducing the subpulse emission features, but is adequate for investigating their drifting behaviour.

We have setup a system of sparks of equal size and separation between adjacent sparks given as $\theta_{s p}=0.2 \theta_{p}$, where $\theta_{p}$ is the angular radius of the non-dipolar polar cap. The sparks resemble a Gaussian shape,

$I_{s p}=I_{0} \exp \left(-4 r^{2} / a_{s p}^{2}\right)$,

where $I_{0}$ is the maximum intensity, $a_{s p}=R_{S} \theta_{s p}$ and $r$ is 

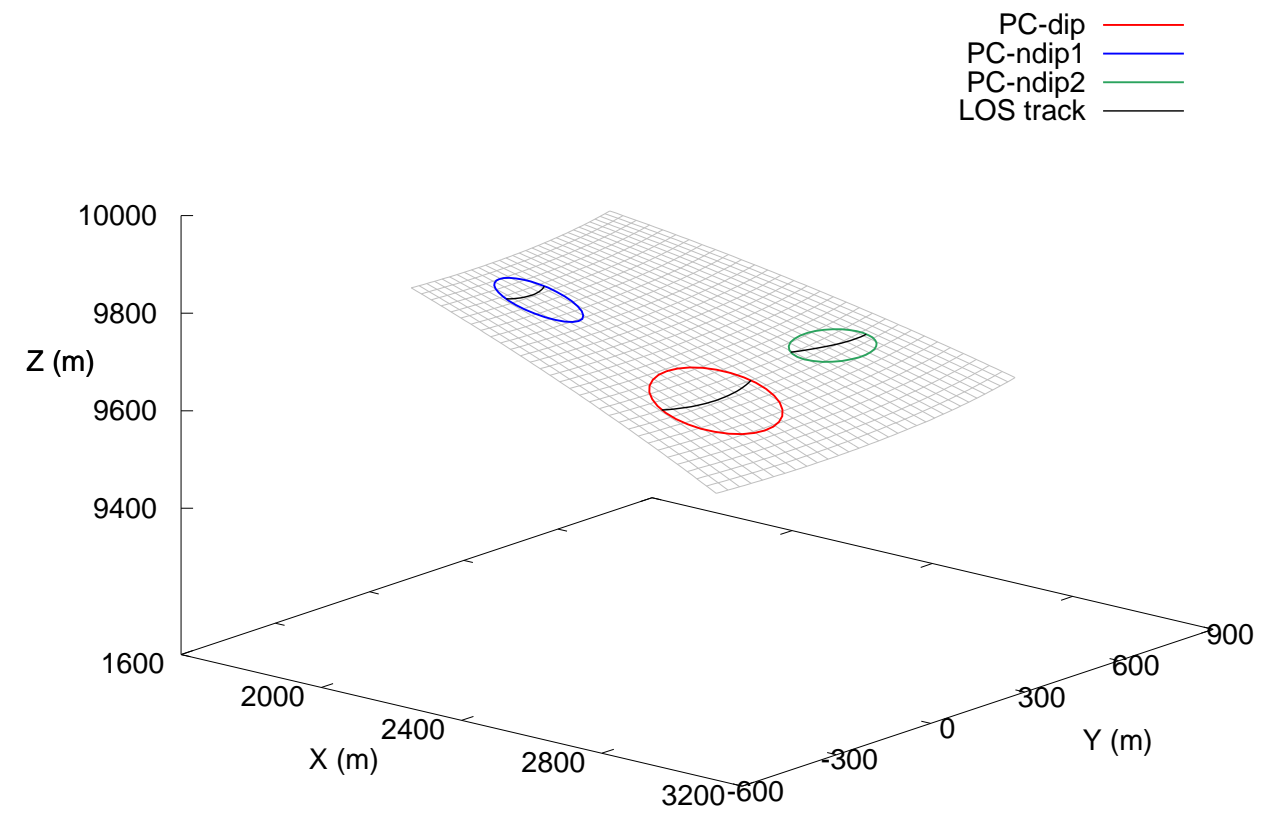

Figure 4. The figure shows three different polar cap orientation for a pulsar with $P=1$ sec and the star centered dipole $\mathbf{d}=\left(d, 15^{\circ}\right.$, $0^{\circ}$ ). The dipolar polar cap is shown in addition to two different realization of non dipolar polar caps. In each of the non-dipolar polar caps one single surface dipole oriented along the z-axis $\mathbf{m}_{s}=\left(0.001 d, 0^{\circ}, 0^{\circ}\right)$ is located at $\mathbf{r}_{s}=\left(0.95 R_{S}, 10^{\circ}, 0^{\circ}\right)$ and $\left(0.95 R_{S}, 15.8^{\circ}\right.$, $18.7^{\circ}$ ) respectively. In addition the projections of the line of sight (LOS), corresponding to $\beta=0^{\circ}$, from the emission region to the polar cap in each of the non dipolar cases are also shown.

given as

$r=\sqrt{d^{2}+d_{i}^{2}-2 d_{i} d\left(\sin \theta \sin \theta_{i} \cos \left(\phi-\phi_{i}\right)+\cos \theta \cos \theta_{i}\right)}$.

Here $(d, \theta, \phi)$ specifies the reference point and $\left(d_{i}, \theta_{i}, \phi_{i}\right)$ the center of the $i^{t h}$ spark. All points considered are on the surface of the neutron star, hence, $d=d_{i}=R_{S}$ and eq.(10) simplifies to the form

$r=1.4 R_{S} \sqrt{1-\left(\sin \theta \sin \theta_{i} \cos \left(\phi-\phi_{i}\right)+\cos \theta \cos \theta_{i}\right)}$.

In the dynamic sparking system a number of spark tracks are setup within the IAR separated by $\theta_{s p}$. In each of these tracks the sparks move around the rotation axis (implying constant $\theta$, since rotation axis is aligned with the z-axis) with the drifting periodicity $P_{3}$. The track locations are identified with $\theta^{n}, n$ representing the track number, which are setup such that a central track passes through the middle of the IAR, $\theta^{0}$, as well as tracks on either side, $\theta^{n}=\theta^{0}+2 n \theta_{s p}, n$ $=0, \pm 1, \pm 2$. At any given time, $t$, the center of the $i^{t h}$ spark is located at $\left(R_{S}, \theta^{n}, \phi_{i}^{n}(t)\right)$,

$\phi_{i}^{n}(t)=2 \pi\left(t-t_{0}\right) / N P_{3}+2 \pi i / N$.

Here $N=\pi \sin \theta^{n} / \theta_{s p} ; i=0,1, \ldots, N-1$ and $t_{0}$ is any arbitrary start time. Using the above setup the time evolution of the sparking process in the IAR from the lagging behind model can be simulated as

$I(t)=\sum_{n} B_{n}(\phi) \sum_{i=0}^{N-1} I_{s p}(t, n, i)$.
$B_{n}(\phi)$ is a box function with $B_{n}(\phi)=1$ for $\phi_{\min }^{n}<\phi<$ $\phi_{\max }^{n}$ and 0 otherwise. Here $\phi_{\min }^{n}$ and $\phi_{\max }^{n}$ are the boundaries of the polar cap along the $n^{\text {th }}$ spark track.

In figure 5 the tracks of the spark motion in the nondipolar polar caps for two different configurations of the magnetic field (corresponding to figure 4) are shown. Additionally, the locus of the LOS variation on these polar caps corresponding to $\beta=0^{\circ}$ are also shown in figure 4 . Depending on the orientation of the surface fields the polar cap is shifted from the corresponding dipolar case. Thus the LOS can traverse the spark tracks at different angles, sometimes across multiple tracks as seen in the right panel of figure 5. This will lead to different phase behaviours in subpulse drifting.

\section{SIMULATING PULSE SEQUENCE FOR SUBPULSE DRIFTING}

In this section we demonstrate that the simplified algorithm presented above can be used to explain the different drifting classes seen in pulsars. To show this in detail we have explored different orientations of the surface dipole to form diverse realisations of the non-dipolar polar cap. In each of these magnetic field configurations we have generated a series of single pulses from sparks lagging behind the corotation speed. This is achieved by rotating the LOS around the star such that $\theta_{E}=\theta_{d}+\beta$ and $\phi_{E}=2 \pi t / P$. When the LOS encounters the open field line region a corresponding translation is made to the IAR by solving the field line equations 

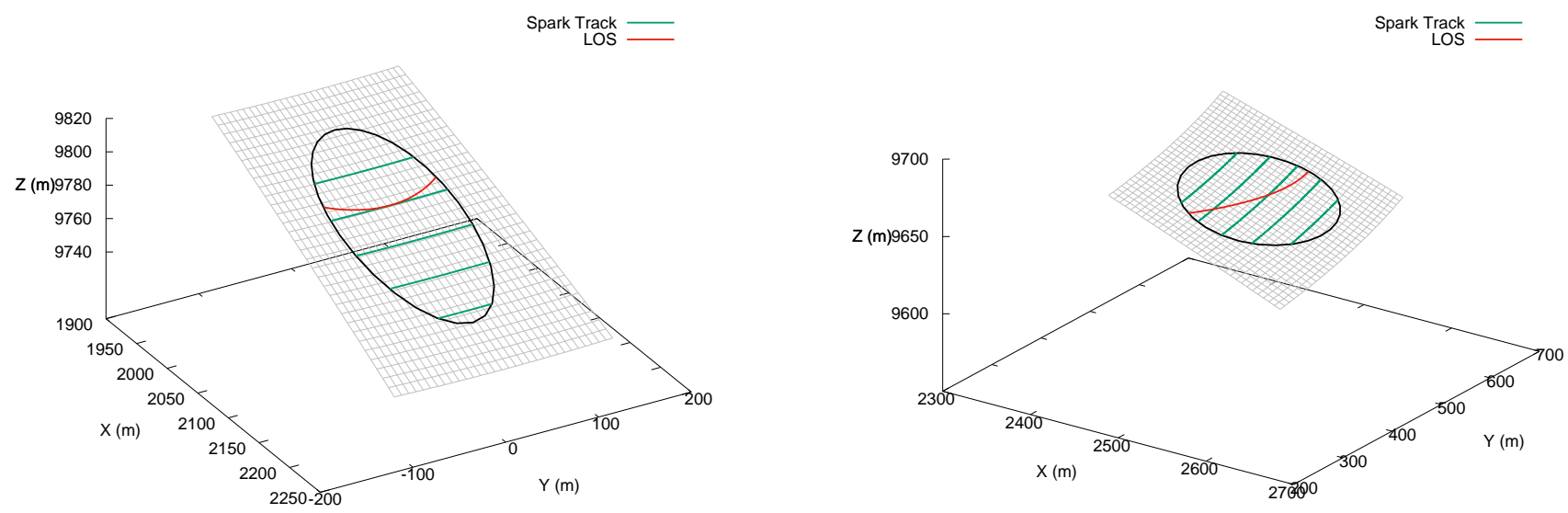

Figure 5. The figure shows track of the sparks which are lagging behind corotation in the Inner Acceleration Region (IAR) of pulsars. In addition the transition of the line of sight (LOS) from the emission region to the polar cap is also shown. The magnetic field structure is governed by two dipoles, the first located at the star center with $\mathbf{d}=\left(d, 15^{\circ}, 0^{\circ}\right)$ and the second near the crust with $\mathbf{m}_{s}=(0.001 d$, $\left.0^{\circ}, 0^{\circ}\right)$ and located at $\mathbf{r}_{s}=\left(0.95 R_{S}, 10^{\circ}, 0^{\circ}\right)$ for the left panel and $\mathbf{r}_{s}=\left(0.95 R_{S}, 15.8^{\circ}, 18.7^{\circ}\right)$ for the right panel. In the right panel it can be seen that the LOS cuts across multiple tracks of spark motion. This is due to the fact that the non-dipolar IAR is asymmetrically displaced from the purely dipolar polar cap.

(eq.8) with initial condition $\mathbf{r}_{E}=\left(50 R_{S}, \theta_{E}, \phi_{E}\right)$ to obtain the corresponding location $\mathbf{r}_{s}=\left(R_{S}, \theta, \phi\right)$ on the polar cap.

Next the dynamic structure of the spark motion in IAR is estimated using eq.(13) and the reference point $\mathbf{r}_{s}$ to obtain the subpulse behaviour. This process is repeated for a number of cycles of the LOS rotation, each rotation corresponding to a single pulse, and the relevant pulse sequence is generated. Finally, we have carried out fluctuation spectral studies on the simulated pulse sequence to explore the phase behaviour of subpulse drifting. In all IAR configurations explored here we show a detailed calculation of the different physical parameters like surface magnetic field behaviour, the ratio $b$, between the non-dipolar and equivalent dipolar field, corotation electric field, $\rho_{G J}, \cos \alpha_{l}$, drift speed due to corotation, etc., in section 5.1.

Note that in our analysis, we show simulations for radio emission for a given observing frequency arising from the same emission height across the line of sight. While this is a reasonably good assumption, however if the emission originated from a range of heights (e.g. Gangadhara 2004), then one expects to see minor changes in the phase behaviour of the drifting subpulse, although the drifting periodicity i.e. $P_{3}$ would remain the same. This effect of phase change arises because for a given pulsar the line of sight remains constant, however for different emission heights the observer cuts different sets of magnetic field lines thus sampling slightly different part of the spark motion in the polar cap. Since this is a purely geometrical effect, there is no change in the repetition time i.e. $P_{3}$. Similar effects of phase change and constant $P_{3}$ would also be seen in observations at different frequencies, since according to radius to frequency mapping progressively higher and higher frequencies arises closer to the neutron star (see e.g. Mitra \& Rankin 2002). Additionally, there is possibility of around $10 \%$ variation in $v_{D}$ and $\cos \alpha_{l}$ across the LOS in certain magnetic field configurations (see section 5.1), which will likely cause $P_{3}$ to vary along the LOS. It is possible to carry out detailed es- timations of the variations of $P_{3}$ along the LOS, but these involve detailed calculations and any estimated variations are expected to be well within the measurement errors in $P_{3}$. As a result we have considered a constant value of $P_{3}$ across the LOS. There is the possibility of certain magnetic field configurations resulting in large change of both $v_{D}$ and $\cos \alpha_{l}$ across the polar cap and as a result the drifting speed is also expected to show large variations across it. However, most observations suggest that $P_{3}$ is mostly constant across the pulsar profile and show very little variations (Weltevrede et al. 2006; Basu et al. 2016, 2019a). Hence, any surface field configuration showing large variations in $v_{D}$ and $\cos \alpha_{l}$ are not viable candidates for these drifting studies.

\subsection{Coherent phase-modulated Drifting}

The coherent phase-modulated drifting is the most prominent drifting class which shows regular drift bands in the single pulse sequence. This can be further divided into two distinct types, positive and negative drifting. The subpulses in such cases show systematic shift in position across the pulse window, with the shift being towards the leading part in case of positive drifting and towards the trailing part in negative drifting. They are also seen as two distinct phase behaviours associated with the peak frequencies in the longitude resolved fluctuation spectral analysis (LRFS, Backer 1973). In case of positive drifting the phases exhibit a systematic variation with a negative gradient from the leading to the trailing edge while the slope is reversed in the case of negative drifting. Basu et al. (2016) argued that the positive and negative drifting classes are due to aliasing of $P_{3}$ around $2 P$. The sparks in the IAR lag behind corotation speed which is characterised by the period of pulsar rotation, i.e, $\Omega=2 \pi / P$. Hence, within the pulse window the sparks move from the trailing to the leading edge as the LOS cuts the emission beam from the leading to the trailing side. Our observations are limited by the fact that the emission is only 

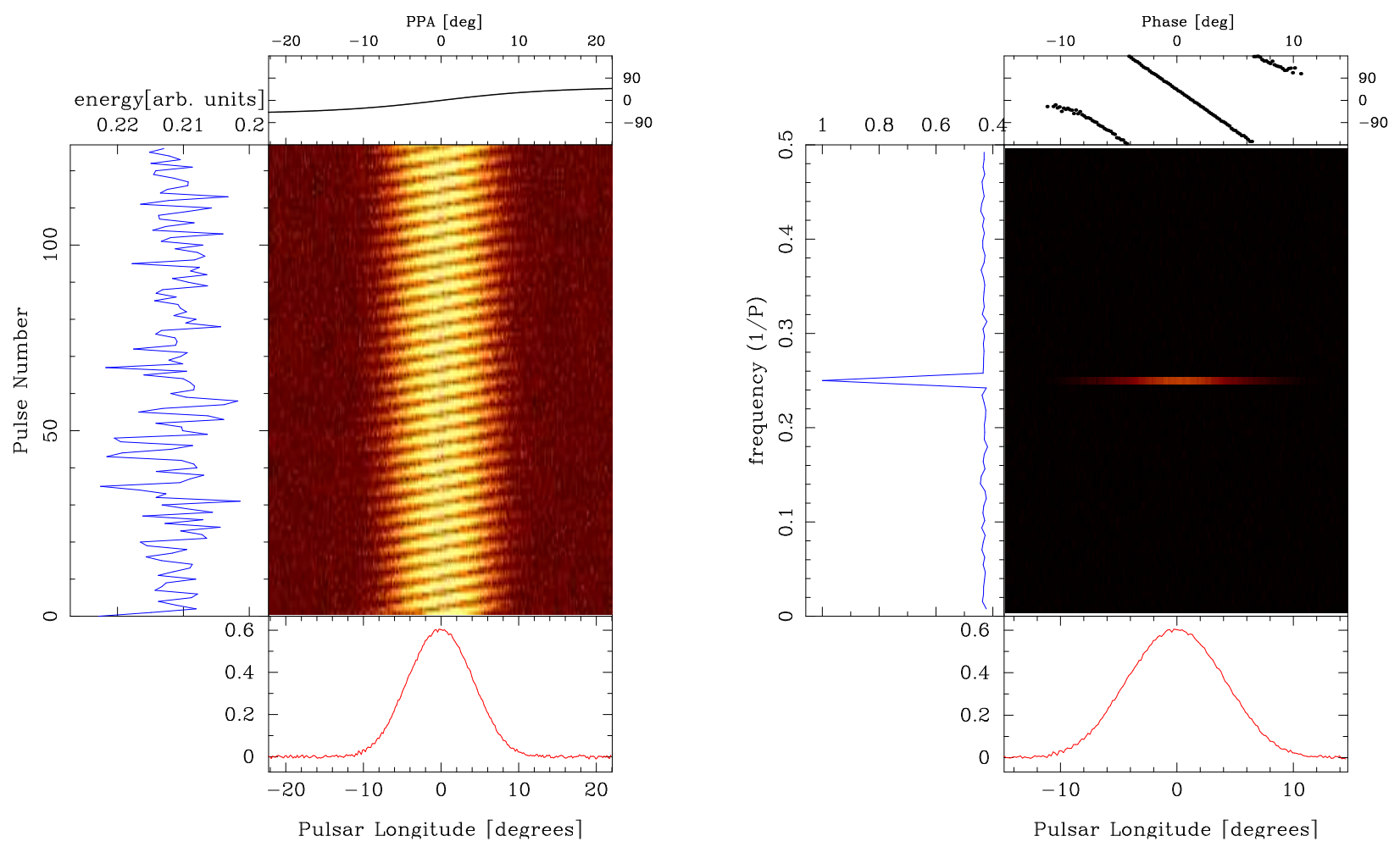

Figure 6. The figure shows a simulation of of a sequence of pulses exhibiting coherent phase-modulated positive drifting. The left panel shows 128 consecutive single pulses where the subpulses in subsequent pulses appear at later longitudes across the pulse window. The right panel shows the LRFS for this pulse sequence which exhibits a peak at $f_{p}=0.25$ cycles $/ P$ and the corresponding phase changes showing a negative slope from the leading to the trailing edge of the window. The pulse sequence was simulated using $P_{3}=1.33 P$. The surface magnetic fields were specified as $\mathbf{r}_{s}=\left(0.95 R_{S}, 10^{\circ}, 0^{\circ}\right)$ and $\mathbf{m}_{s}=\left(0.001 d, 0^{\circ}, 0^{\circ}\right)$. The inclination of star centered dipole was $\theta_{d}=15^{\circ}$ and the line of sight inclination angle $\beta=4^{\circ}$.

PC-Coh Pos $=$

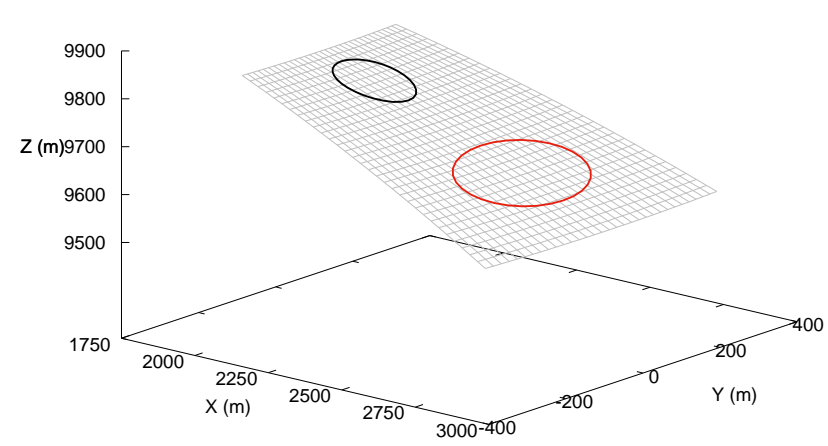

Spark Track $=$

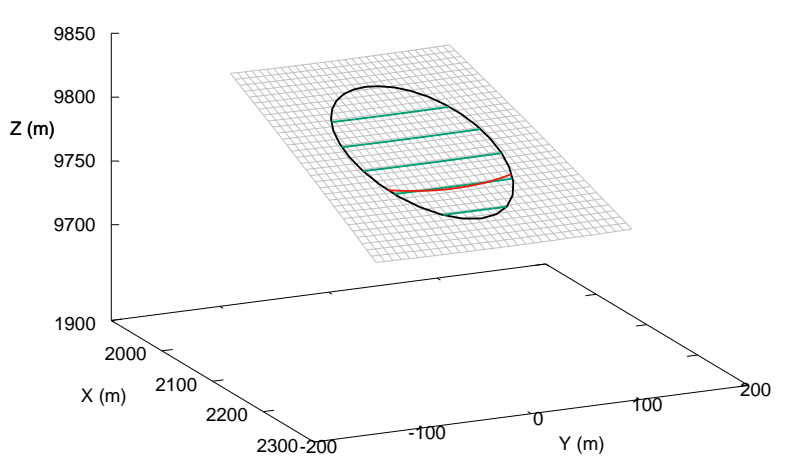

Figure 7. The figure shows location of the non-dipolar polar cap with respect to an equivalent dipolar case (left panel) and the tracks of the sparks (right panel) which are lagging behind corotation in the Inner Acceleration Region (IAR). In addition the transition of the line of sight (LOS) from the emission region to the polar cap is also shown. The magnetic field is characterised by two dipoles, one located at the star center with $\mathbf{d}=\left(d, 15^{\circ}, 0^{\circ}\right)$ and the second near the crust with $\mathbf{m}_{s}=\left(0.001 d, 0^{\circ}, 0^{\circ}\right)$ located at $\mathbf{r}_{s}=\left(0.95 R_{S}, 10^{\circ}\right.$, $\left.0^{\circ}\right)$. The LOS corresponds to $\beta=4^{\circ}$ and closely follows the spark track. 

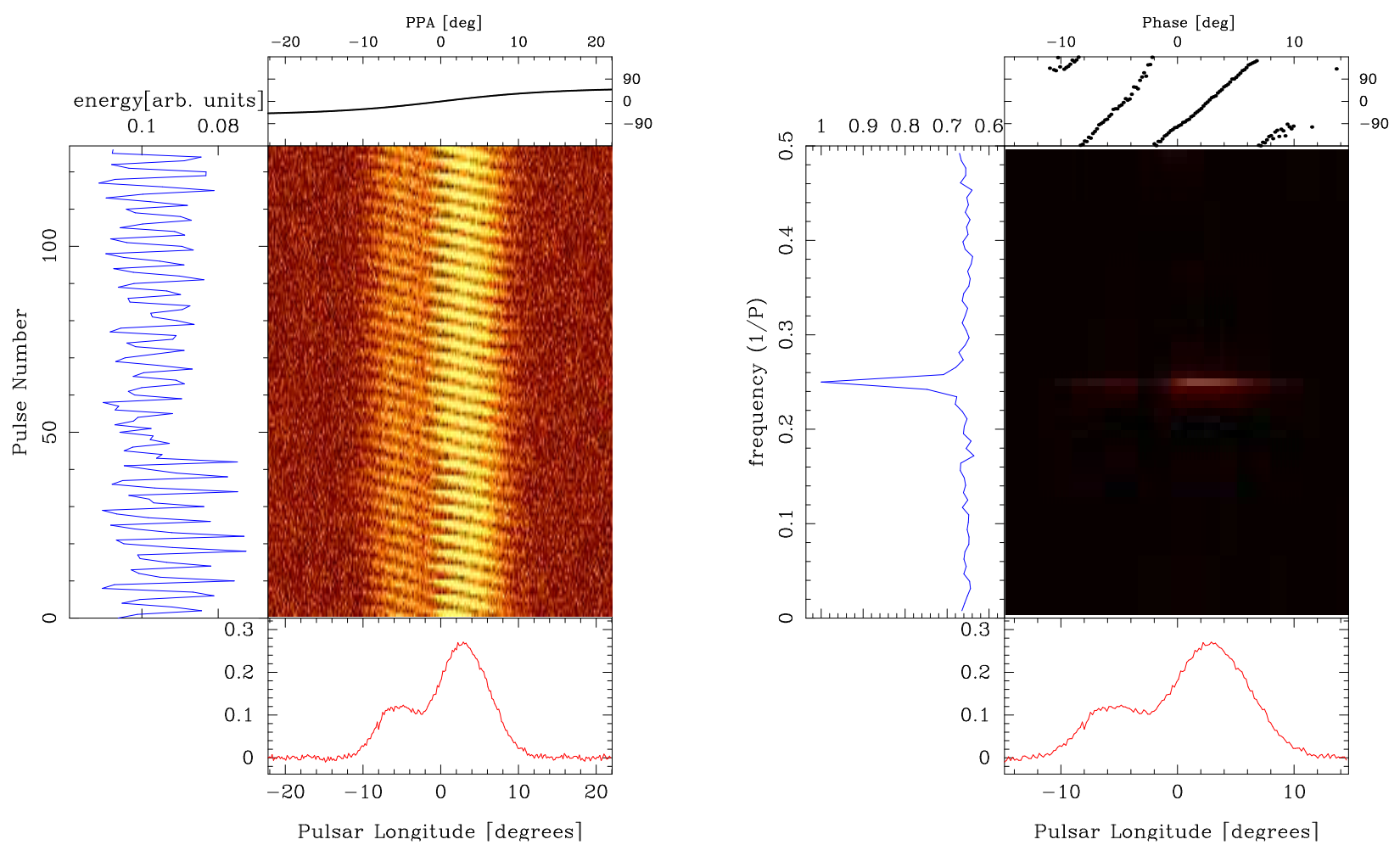

Figure 8. The figure shows a simulation of a sequence of pulses exhibiting coherent phase modulated negative drifting. The left panel shows 128 consecutive single pulses where the subpulses in subsequent pulses appear at earlier longitudes across the pulse window. The right panel shows the LRFS for this pulse sequence which exhibits a peak at $f_{p}=0.25 \mathrm{cycles} / P$ and the corresponding phase changes showing a positive slope from the leading to the trailing edge of the window. The pulse sequence was simulated using $P_{3}=4 P$. The surface magnetic fields were specified as $\mathbf{r}_{s}=\left(0.95 R_{S}, 18.86^{\circ}, 10.99^{\circ}\right)$ and $\mathbf{m}_{s}=\left(0.001 d, 0^{\circ}, 0^{\circ}\right)$. The inclination of star centered dipole was $\theta_{d}=15^{\circ}$ and the line of sight inclination angle $\beta=4^{\circ}$.
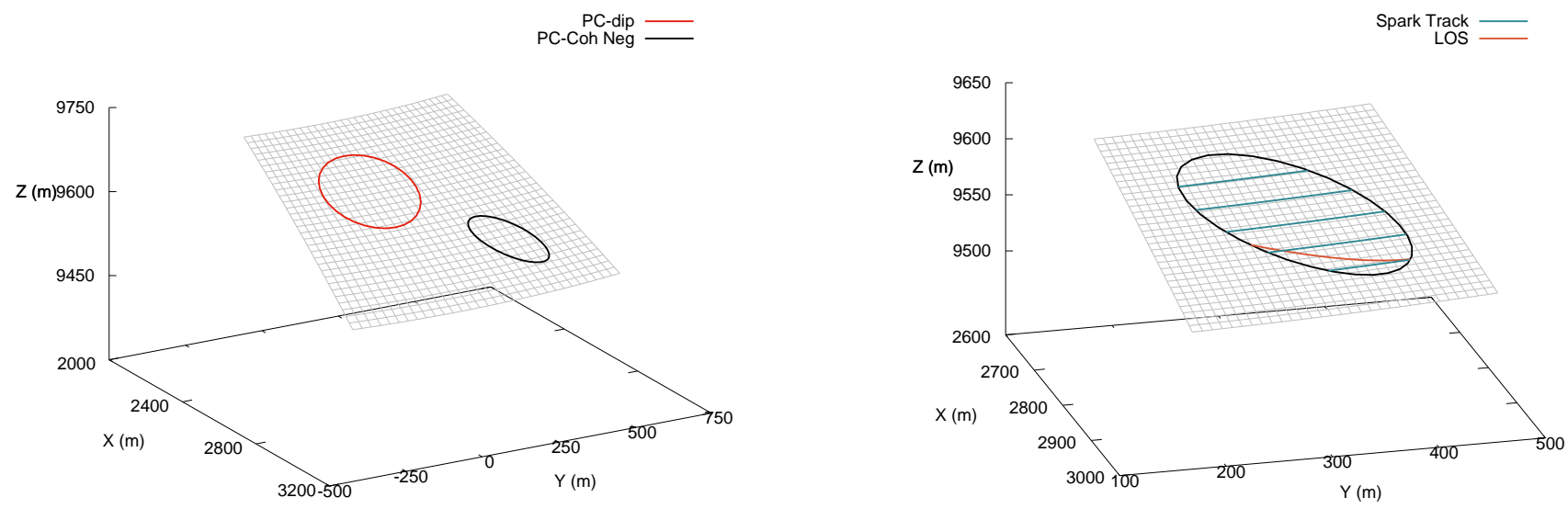

Figure 9. Equivalent to figure 7 for the magnetic field configuration $\mathbf{d}=\left(d, 15^{\circ}, 0^{\circ}\right)$ and $\mathbf{m}_{s}=\left(0.001 d, 0^{\circ}, 0^{\circ}\right)$ located at $\mathbf{r}_{s}=\left(0.95 R_{S}\right.$, $\left.18.86^{\circ}, 10.99^{\circ}\right)$. 
seen once every period which implies that we can only see features below $f_{p}<0.5$ cycles $/ P$ in the LRFS. So, if the drifting periodicity $P_{3}>2 P$ the subpulses in subsequent periods appear at earlier longitudes which is the negative drifting. However, if the drifting periodicity is $P<P_{3}<$ $2 P$, the subpulses in every subsequent period would appear at later longitudes and correspond to the positive drifting. The measured peak frequency in the LRFS in this case is aliased and corresponds to $f_{p}=1-P / P_{3}$. Here, the underlying assumption is $P_{3}<P$ is not a viable solution, hence the frequency peaks are not associated with higher order aliases.

In figure 6 we show one realisation of positive drifting. The left panel shows 128 single pulses where the subpulses shift towards the trailing part of the profile. The right panel shows the LRFS corresponding to this pulse sequence. The magnetic field configuration comprises of a star centered dipole with $\theta_{d}=15^{\circ}$ and the surface dipole with $\mathbf{m}_{s}$ $=\left(0.001 d, 0^{\circ}, 0^{\circ}\right)$ located at $\mathbf{r}_{s}=\left(0.95 R_{S}, 10^{\circ}, 0^{\circ}\right)$. We have used $\beta=4^{\circ}$ for simulating the single pulses with the average profile showing a single component. The polar cap structure is represented in figure 7 , which shows the different spark tracks in the IAR as well as the LOS traverse across them. The magnetic field configuration in this case is symmetric around the y-axis. This is reflected in the LOS traverse which closely follow a single spark track. This is also seen in the phase variations associated with the drifting peak which is linear, apart from the edges which show a slight flattening due to the LOS being more curved than the spark tracks. We have used $P_{3}=1.33 P$ for generating the subpulse motion which corresponds to positive drifting and is seen as the drifting peak at $f_{p}=0.25$ cycles $/ P$ in the LRFS. The corresponding negative drifting for this configuration can be produced using $P_{3}=4 P$ which will give identical $f_{p}$ with the sign of the phase slope reversed.

Another realisation of a single pulse sequence with prominent drift bands is shown in figure 8 . In this case we have reproduced negative drifting with the subpulses shifting towards the leading part of the profile (left panel). We have used $P_{3}=4 P$ for the spark motion in the IAR which once again gives a peak around $f_{p}=0.25$ cycles $/ P$ in the LRFS (right panel). But contrary to the previous case the magnetic field configuration is more asymmetric with the surface dipole once again given as $\mathbf{m}_{s}=\left(0.001 d, 0^{\circ}, 0^{\circ}\right)$ but located at $\mathbf{r}_{s}=\left(0.95 R_{S}, 18.86^{\circ}, 10.99^{\circ}\right)$. We have once again used $\beta=4^{\circ}$ for simulating the single pulses. The polar cap structure for this configuration is shown in figure 9 where the asymmetry is reflected in the elongated polar cap shape as well as the LOS traverse. The LOS cuts across two spark tracks resulting in a barely resolved double peaked structure. It should also be noted that the phase variations associated with the drifting (figure 8, right panel, top window) show distinct non-linear behaviour. This is more representative of the phase behaviour seen in pulsar observations (Basu et al. 2016).

\subsection{Low-mixed phase-modulated Drifting}

The second category of subpulse drifting investigated here belongs to the low-mixed phase-modulated drifting, where the subpulses do not show much change in their relative location within the pulse window but periodically vary in intensity. This is usually associated with profiles which show multiple components, implying that the LOS cuts the emission beam more centrally with low $\beta$ (Basu et al. 2019a). We have reproduced an example of this phenomenon as shown in figure 10. The left panel shows 128 consecutive single pulses with three subpulses which do not move across the profile but periodically varies in intensity, forming a three component average profile. We have used a magnetic configuration specified by a star centered dipole with $\theta_{d}=45^{\circ}$ and another surface dipole with $\mathbf{m}_{s}=\left(0.05 d, 0^{\circ}, 0^{\circ}\right)$ located at $\mathbf{r}_{s}$ $=\left(0.95 R_{S}, 57.08^{\circ}, 20.66^{\circ}\right)$. The $\beta$ used for LOS estimates was $0.1^{\circ}$. The right panel of the figure shows the LRFS for this pulse sequence. We have use $P_{3}=3.33 P$ for spark motion in IAR which is reflected as the frequency peak $f_{p}=$ 0.3 cycles $/ P$ in the LRFS. The phase variations in the top window of the right panel show very little change across each component, but exhibit jumps between adjacent components which is consistent with observations of low-mixed phasemodulated drifting (Basu et al. 2019a). Figure 11 shows the conditions in the polar cap corresponding to the magnetic configuration. The small shifts of the subpulse position suggests that the LOS should traverse across the paths of spark motion. This is clearly seen in the right panel of the figure where the LOS is indeed cutting across the tracks. To achieve this arrangement the non-dipolar polar cap had to be shifted significantly away from the dipolar counterpart as seen in the left panel of the figure.

\subsection{Switching phase-modulated Drifting}

The switching phase-modulated drifting is seen in a small group of pulsars where the subpulses belonging to different components of the profile show large shifts and reversals in phase directions. This implies that both positive and negative drifting are seen at different regions of the pulsar profile. In figure 12 we show an example of the switching phase-modulated drifting behaviour using sparks lagging behind corotation speed. The left panel shows 128 single pulses where subpulses show the gradual reversal in phase from the first to the last component. The pulsar profile consists of four components with significant subpulse variation across each of them. The leading component has the most variation and shows negative drifting with shifts towards the leading part of the profile. The two central components has lower variations in phases with the central left component showing slight negative drifting and the central right component small positive drifting. The trailing component shows positive drifting with the subpulses shifting towards the trailing edge. The magnetic configuration used in these simulations consists of a star centered dipole with $\theta_{d}=5^{\circ}$ and another surface dipole with $\mathbf{m}_{s}=\left(0.005 d, 0^{\circ}, 0^{\circ}\right)$ located at $\mathbf{r}_{s}=$ $\left(0.95 R_{S}, 5^{\circ}, 120^{\circ}\right)$. We have used $\beta=0.1^{\circ}$ for the LOS. The right panel of the figure shows the LRFS corresponding to the single pulse behaviour. We have used $P_{3}=10 P$ for the spark motion in the IAR which is reflected as the peak frequency $f_{p}=0.1$ cycles $/ P$ in the LRFS. The top window also shows the phase behaviour which indicates the bi-drifting behaviour.

Figure 13 shows the conditions in the IAR which leads to switching phase-modulated drifting. The LOS makes a curved traverse across the different spark paths. Initially the LOS moves in the opposite direction of the spark motion which results in negative drifting. For the two central tracks 

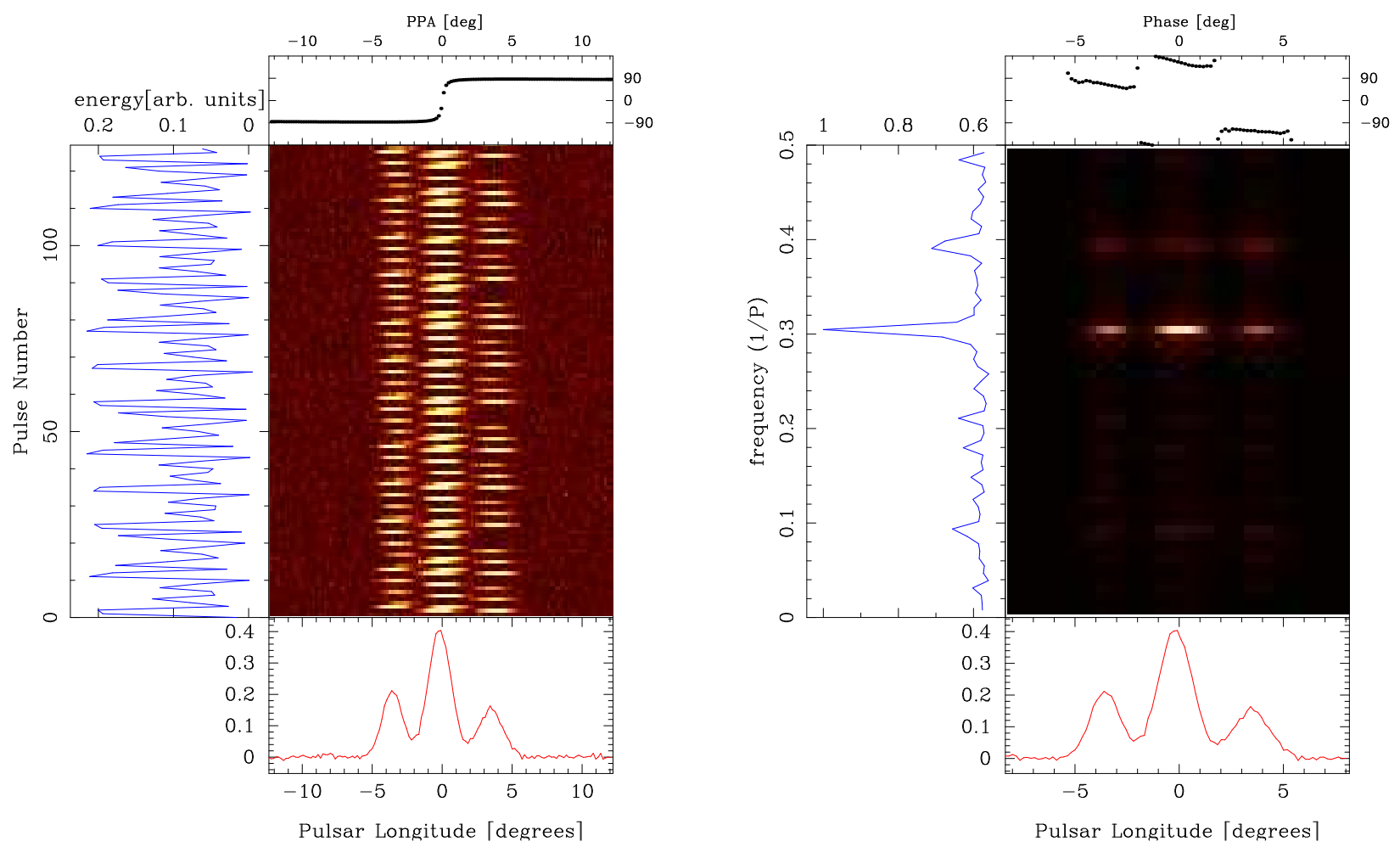

Figure 10. The figure shows a simulation of a sequence of pulses exhibiting low-mixed phase-modulated drifting. The left panel shows 128 consecutive single pulses where the subpulses in subsequent pulses do not move steadily across the pulse window but vary periodically in intensity. The right panel shows the LRFS for this pulse sequence which exhibits a peak at $f_{p}=0.3$ cycles $/ P$ and the corresponding phase changes across each component are relatively flat. The pulse sequence was simulated using $P_{3}=3.33 P$. The surface magnetic fields were specified as $\mathbf{r}_{s}=\left(0.95 R_{S}, 57.08^{\circ}, 20.66^{\circ}\right)$ and $\mathbf{m}_{s}=\left(0.05 d, 0^{\circ}, 0^{\circ}\right)$. The inclination of star centered dipole was $\theta_{d}=45^{\circ}$ and the line of sight inclination angle $\beta=0.1^{\circ}$.

$\underset{\text { PC-Low Mix }}{\text { PC-ip }}$

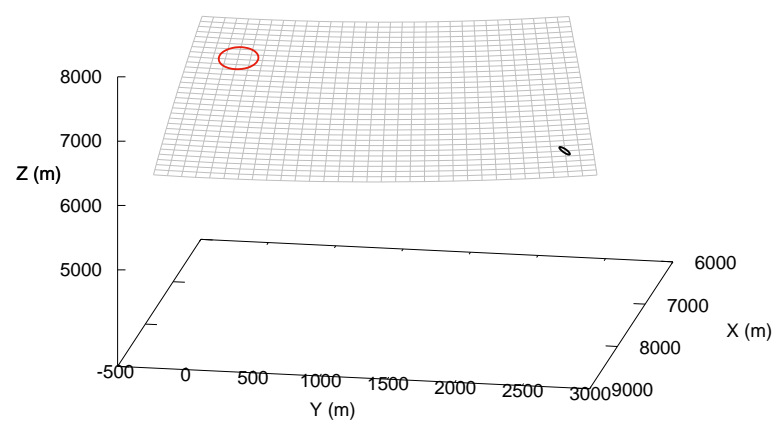

Spark Track

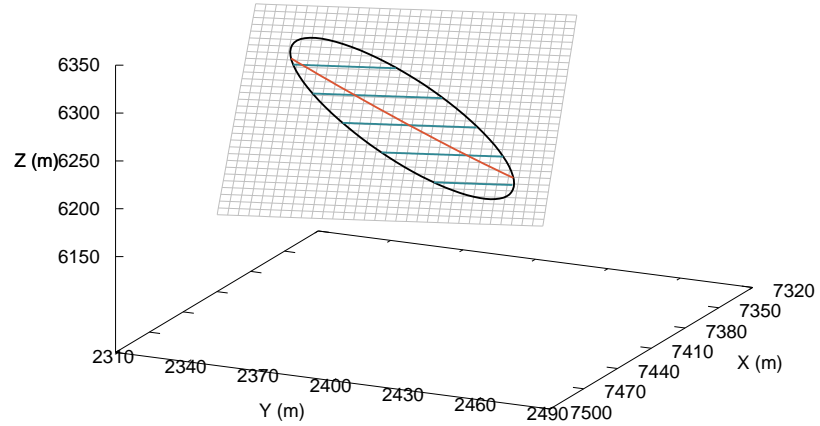

Figure 11. Equivalent to figure 7 for the magnetic field configuration $\mathbf{d}=\left(d, 45^{\circ}, 0^{\circ}\right)$ and $\mathbf{m}_{s}=\left(0.05 d, 0^{\circ}, 0^{\circ}\right)$ located at $\mathbf{r}_{s}=\left(0.95 R_{S}\right.$, $\left.57.08^{\circ}, 20.66^{\circ}\right)$. The LOS corresponds to $\beta=0.1^{\circ}$ and cuts across the spark tracks. 

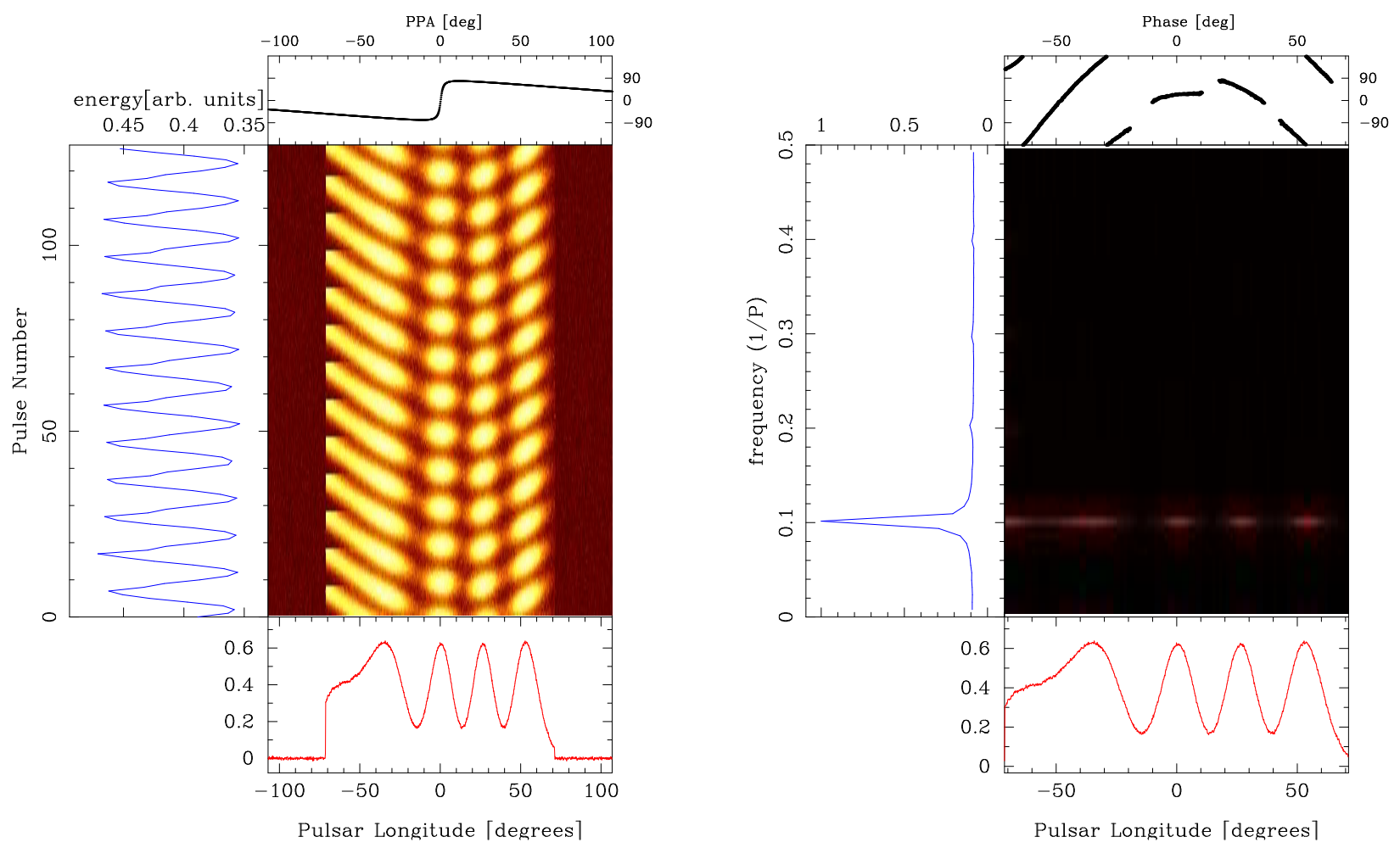

Figure 12. The figure shows a simulation a sequence of pulses exhibiting switching phase-modulated drifting where the drift direction is opposite in different parts of the profile. The left panel shows 128 consecutive single pulses where the subpulses in the leading part of the profile appear at earlier longitudes while the subpulses near the trailing edge appear at later longitudes in subsequent pulses. The right panel shows the LRFS for this pulse sequence which exhibits a peak at $f_{p}=0.1$ cycles $/ P$ and the corresponding phases show a positive slope in the leading part of the profile which becomes flatter near the middle and changes to negative slope in the trailing part. The pulse sequence was simulated using $P_{3}=10 P$. The surface magnetic fields were specified as $\mathbf{r}_{s}=\left(0.95 R_{S}, 5^{\circ}, 120^{\circ}\right)$ and $\mathbf{m}_{s}=$ $\left(0.005 d, 0^{\circ}, 0^{\circ}\right)$. The inclination of star centered dipole was $\theta_{d}=5^{\circ}$ and the line of sight inclination angle $\beta=0.1^{\circ}$.

PC-dip
PC-Swi Phs

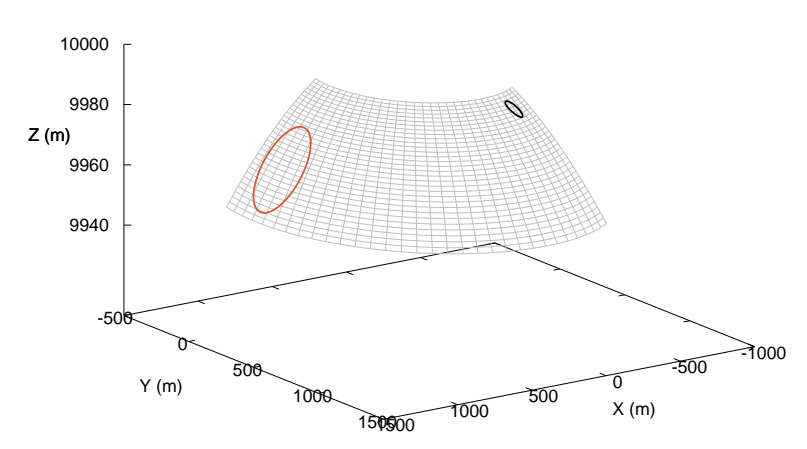

Spark Track —

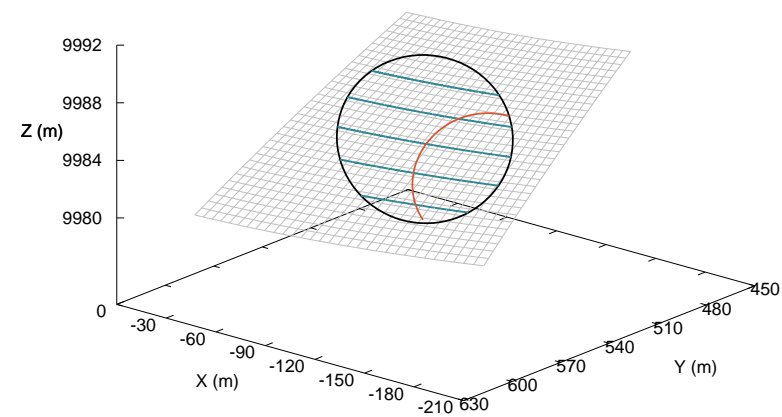

Figure 13. Equivalent to figure 7 for the magnetic field configuration $\mathbf{d}=\left(d, 5^{\circ}, 0^{\circ}\right)$ and $\mathbf{m}_{s}=\left(0.005 d, 0^{\circ}, 0^{\circ}\right)$ located at $\mathbf{r}_{s}=\left(0.95 R_{S}\right.$, $\left.5^{\circ}, 120^{\circ}\right)$. The LOS corresponds to $\beta=0.1^{\circ}$ and cuts the leading and trailing parts at opposite directions of spark motion leading to the bi-drifting phenomenon. 
the LOS cuts across them resulting in very little variations across the components. Finally, towards the trailing side the LOS reverses direction and moves in the same direction of the sparks which results in positive drifting. The left panel in the figure shows that the non-dipolar polar cap has to be highly asymmetric compared to the equivalent dipolar polar cap in order to see the bi-drifting behaviour. The polar cap is shifted to the other side of the neutron star rotation axis (negative $\mathrm{x}$-direction) resulting in highly curved nature of the LOS in this example. This further explains the rarity of this phenomenon in the pulsar population.

Also note that the $\theta_{d}=5^{\circ}$ is the lowest amongst the different configurations explored in this work. This has important observational implications. In all pulsars exhibiting reversals in phases the profile width is much larger than the general pulsar population $\left(>50^{\circ}\right.$, see Basu et al. 2019a,b), which suggests a low value of magnetic inclination angle, $\theta_{d}$. In our simulations the reversals in phase is possible because the non-dipolar polar cap is rotated to the other side of the neutron star. In case the inclination angle is large the surface field needs to be significantly larger on the other side for this realization. This is difficult to achieve in a realistic pulsar where there are likely to be multiple surface anomalies resulting in strong local fields, and the nearest maximum to the dipolar polar cap dominating the eventual IAR. Hence, the relatively wide profiles in bi-drifting pulsars provide indirect evidence for the sparks to lag behind corotation speed in IAR dominated by non-dipolar fields.

\section{DISCUSSION}

The different drifting classes can be associated with the differential orientation of the non-dipolar polar cap compared to the corresponding dipolar case. The systematic drift bands corresponding to coherent phase-modulated drifting are seen when the polar cap orientation is close to the dipolar case, i.e, the dominating surface dipoles are close to the magnetic axis. As the surface dipoles are oriented further away from the axis the systematic drift bands give way to non-linearity in the phase variations till phase stationary behaviour is seen when the non-dipolar polar cap is on the sides of the neutron star relative to the dipolar case, i.e. roughly $90^{\circ}$ away. Finally, the reversals in phase behaviour is seen when the surface magnetic field is highly curved and the LOS reverses direction as it traverses the polar cap. This is usually seen when the non-dipolar polar cap is rotated behind the dipolar polar cap, i.e. almost $180^{\circ}$ away. There is increasing evidence for the presence of non-dipolar polar caps in normal pulsars $(P>0.1 \mathrm{~s})$ from simultaneous observations at radio and $\mathrm{X}$-ray frequencies. The thermal X-ray peak, which originates from the heated polar cap, and the radio emission peak, arising from the dipolar field lines are significantly misaligned (Arumugasamy \& Mitra 2019; Pétri \& Mitra 2020). This is only possible when the polar cap is highly non-dipolar in nature and located far away from the corresponding dipolar case. Additionally, other observed correlations, like bi-drifting behaviour being associated with relatively wide profile pulsars, the constant drifting periodicity across all components of the profile (see section 5.1), also find simple interpretation in this proposed mechanism. There is no requirement for the sparks to lag behind coro- tation speed in certain parts of the polar cap and exceed corotation speed in other parts, in order to reproduce the different drifting features.

There is also an underlying assumption of steady evolution of sparks, where they freely lag behind the corotation speed throughout the IAR. However, this idealized lagging behind scenario is restricted in reality by the presence of polar cap boundaries beyond which no spark can be formed in the closed field line region. As the the pair production process is inhibited in the closed field line region a continuous sparking discharge is expected to happen near the edge of the polar cap for the thermal regulation in the PSG model. If a spark is not present near the boundary, since there is no heating from the other side, the temperature drops quickly inhibiting the ion flow from the surface. A large potential drop along the IAR develops resulting in sparking discharge to commence immediately near the boundary. Hence, the most likely dynamics of the plasma flow in IAR would involve the sparks to lag behind corotation, but constrained to move along the boundary of the polar cap, such that there is always presence of a spark near the edge. In addition, the PSG model also demands the IAR to be tightly packed with sparks for effective thermal regulation throughout the polar cap. As a result of the boundary effect one expects the spark motion to be increasingly constrained as one moves away from the edge and the sparks are expected to be completely stationary near the central region. A detailed study incorporating the boundary effect on the sparks and their natural tendency to lag behind corotation speed will be explored in a future work. The possibility of stationary sparks near the center has important observational implications. The pulsar emission is characterised by a stationary core which does not show drifting (Rankin 1986; Basu et al. 2019a). In addition, there are also a large number of cases where no subpulse drifting is seen in the single pulse emission (Weltevrede et al. 2006, 2007; Basu et al. 2016, 2019a). In the absence of detailed estimates a simplified application of the effect of stationary sparks in the center of the IAR can be used for the single pulse simulations shown in section 4. The sparks in the central tracks used in all cases can be considered to be stationary with no time evolution, i.e., in eq. (12), $\phi_{i}^{0}(t)=2 \pi i / N$. There is no change in the observed drifting behaviour for the coherent phase-modulated drifting cases in section 4.1, where the LOS traverses the IAR peripherally without cutting across the central spark track. The modified single pulse behaviour corresponding to low-mixed phase-modulated drifting case is shown in figure 14 , and switching phase-modulated drifting is shown in figure 15. The magnetic field configurations and the drifting periodicities used in these plots are identical to section 4.2 and 4.3, respectively. The central component, resembling the core emission, is stationary in both cases without any periodic behaviour.

\subsection{Physical parameters in non-dipolar Inner Acceleration Region}

The estimates of the different physical parameters presented in the appendix A and B, and is applied to the non-dipolar polar cap configurations used in section 4 to explain the different drifting behaviour. These include, the ratio between the non-dipolar surface field with the equivalent dipolar field 

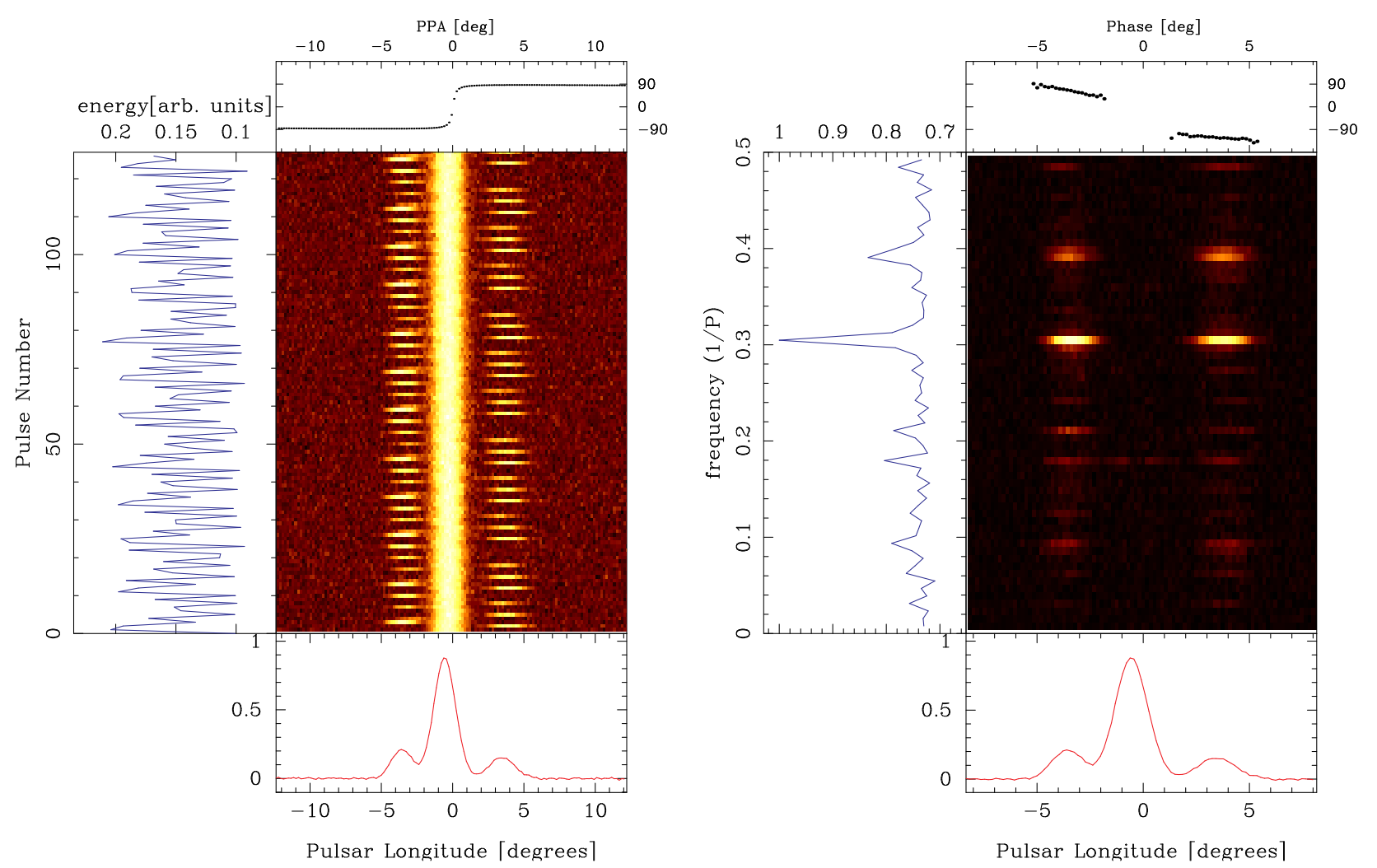

Figure 14. The figure shows the single pulse behaviour for the modified spark motion in the IAR corresponding to the magnetic field configuration shown in figure 11 which results in the low-mixed phase-modulated drifting. In this modified figure the sparks are assumed to be stationary in the central track. The presence of a boundary around the polar cap and tightly packed sparks in the IAR inhibit the spark motion, particularly near the center. The single pulse sequence for 128 pulses is shown in the left panel, where the central component is unchanging while the outer components show periodic modulation in intensity. The right panel shows the LRFS for this pulse sequence which exhibits a peak at $f_{p}=0.3$ cycles $/ P$ in the two outer components with relatively flat phase variations. No periodic fluctuation is seen in the central component.

defined as $b=B_{s} / B_{d}$, the radius of curvature, $\rho_{c}$, shown in appendix $\mathrm{B}$, the ratio between the angular components of the local magnetic field with the radial component, $B_{\theta} / B_{r}$ and $B_{\phi} / B_{r}$, shown in eq.(A1) to eq.(A4), the angle $\cos \alpha_{l}$ between the local magnetic field and the $\mathrm{z}$-axis, and the drift velocity due to corotation, $\mathbf{v}_{D}^{c}$, using eq.(7). In addition, we have also estimated these physical parameters for a purely dipolar polar cap configuration for comparisons, with the exception of estimating $B_{d}$ instead of $b$, which is identically 1 by definition in this case. In figure 16 we present variations of the different quantities across the polar cap for a star centered dipole with inclination angle $\theta_{d}=15^{\circ}$. Figure 17 shows the respective parameters for a non-dipolar polar cap defined by a star centered dipole with $\theta_{d}=15^{\circ}$ and a near surface counterpart located at $\mathbf{r}_{s}=\left(0.95 R_{S}, 18.86^{\circ}\right.$, $\left.10.99^{\circ}\right)$ with magnetic moment $\mathbf{m}_{s}=\left(0.001 d, 0^{\circ}, 0^{\circ}\right)$. This polar cap configuration is responsible for the coherent phasemodulated drifting described in section 4.1. In figure 18 a different non-dipolar polar cap is shown consisting of a star centered dipole with $\theta_{d}=45^{\circ}$ and a near surface counterpart located at $\mathbf{r}_{s}=\left(0.95 R_{S}, 57.08^{\circ}, 20.66^{\circ}\right)$ with magnetic moment $\mathbf{m}_{s}=\left(0.05 d, 0^{\circ}, 0^{\circ}\right)$. This magnetic configuration is used to simulate the low-mixed phase modulated drifting in section 4.2. Finally, in figure 19 we show the variations of the physical parameters for the non-dipolar polar cap corresponding to the switching phase-modulated drift- ing explored in section 4.3. The magnetic field configuration consists of a star centered dipole with $\theta_{d}=5^{\circ}$ and a near surface dipole located at $\mathbf{r}_{s}=\left(0.95 R_{S}, 5^{\circ}, 120^{\circ}\right)$ with magnetic moment $\mathbf{m}_{s}=\left(0.005 d, 0^{\circ}, 0^{\circ}\right)$. All quantities are calculated on the surface of the neutron star with $R_{S}=10 \mathrm{~km}$ and the variations are expressed using a suitable colour scale in the $\mathrm{X}-\mathrm{Y}$ plane, since the fractional extent of the polar cap is least along the z-axis.

Clear differences emerge between the purely dipolar and non-dipolar polar caps from these estimates. In case of the dipolar polar cap the magnetic field show very little variations across the polar cap with less than hundredth of a percentage difference from the edge to the center. The configuration has an extremum at the field center which is circularly symmetric. In contrast the non-dipolar polar caps show much larger variations in $b \sim 50-100 \%$, which changes monotonically from one end of the polar cap to the other. The radius of curvature is also vastly different between the dipolar polar cap and other non-dipolar cases. In the purely dipolar case it shows large variations from the edge of the polar cap $\left(\sim 10^{8} \mathrm{~cm}\right)$ to the central regions and is circularly symmetric around the magnetic axis. In non-dipolar polar caps the radius of curvature varies monotonically from one end to the other with maximum change around 20-30\%. In all cases the estimated $\rho_{c} \sim 10^{5}-10^{6} \mathrm{~cm}$, which is the preferred curvature for efficient pair production in IAR and con- 

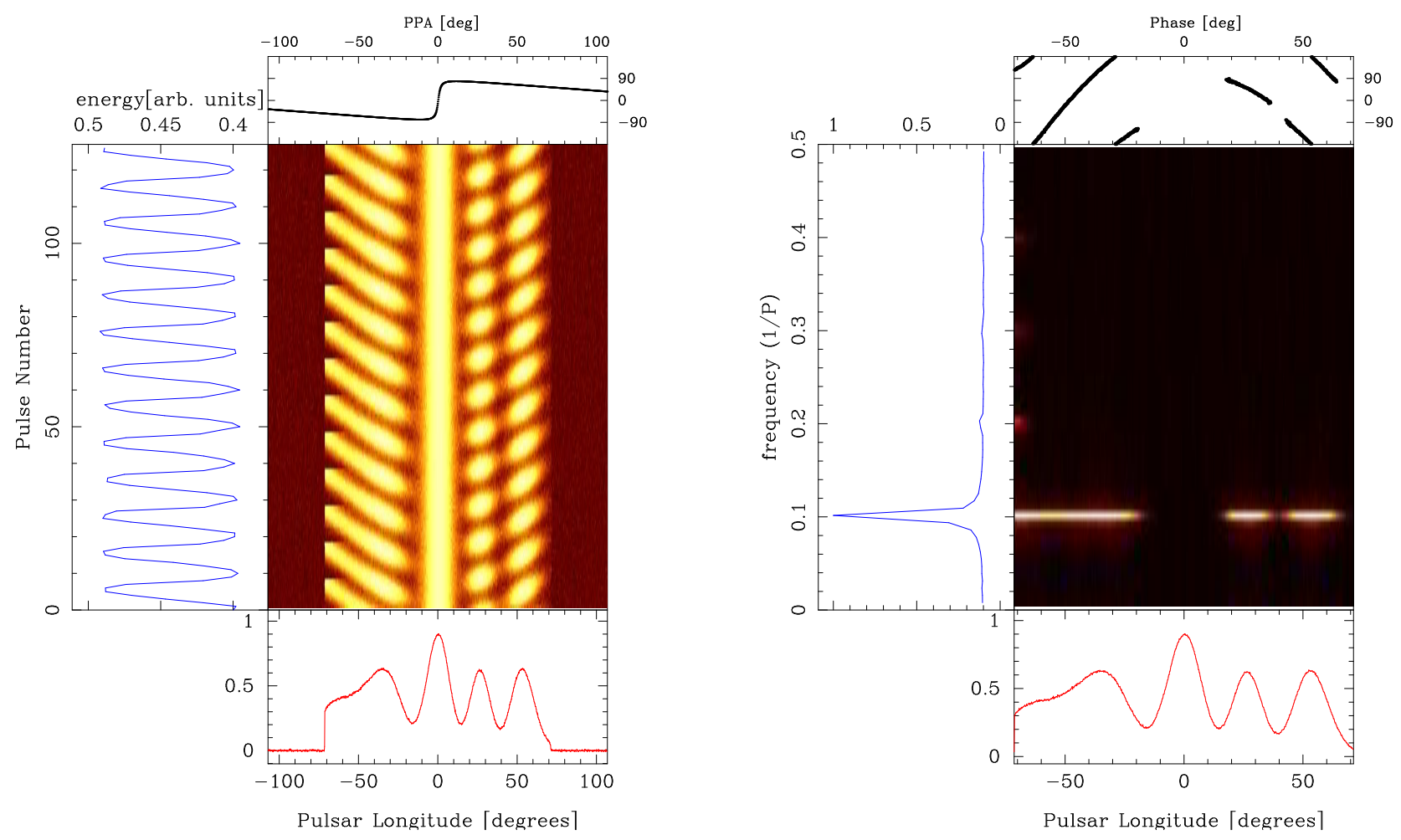

Figure 15. The figure shows the single pulse behaviour for the modified spark motion in the IAR corresponding to the magnetic field configuration shown in figure 13 which results in the switching phase-modulated drifting. In this modified figure the sparks are assumed to be stationary in the central track. The presence of a boundary around the polar cap and tightly packed sparks in the IAR inhibit the spark motion, particularly near the center. The single pulse sequence for 128 pulses is shown in the left panel, where the central component is unchanging while in the outer components the subpulses in the leading part of the profile appear at earlier longitudes while the subpulses near the trailing edge appear at later longitudes in subsequent pulses. The right panel shows the LRFS for this pulse sequence which exhibits a peak at $f_{p}=0.1$ cycles $/ P$ in the outer components and the corresponding phases show a positive slope in the leading part of the profile which transforms to negative slope in the trailing part. No periodic fluctuation is seen in the central component.

sequently the sparking process to be sustained (see RS75). The estimates of the magnetic field strength and $\rho_{c}$ across the non-dipolar polar cap further highlights that the sparking process can commence at any location with identical properties in a realistic polar cap, governed by local thermal conditions of the PSG. The angular components of the magnetic field are much more prominent in case of non-dipolar polar caps, where $B_{\theta} / B_{r}$ and $B_{\phi} / B_{r}$ can be several times the radial component. In contrast the angular components of the magnetic field in the dipolar polar cap is less than $1 \%$ of the radial component. A number of studies (Szary 2013; Mitra et al. 2020) have evaluated the charge motion within the IAR based on analytical estimates, with the assumption that the angular components of the magnetic field is negligible in the IAR. The large contribution of angular components of the magnetic field in non-dipolar polar caps indicate that these analytical estimates of particle motion are inadequate and more detailed simulations are necessary for studying the rigorous evolution of any sparking process. Finally, the estimates of the drift speeds and $\cos \alpha_{l}$ show that although they have large diversity for different magnetic field configurations, they vary around $10 \%$ across any particular polar cap. Thus even in our non-rigorous estimate of sparks lagging behind corotation speed with a fraction of $v_{D}$ specified by $\eta$, the drifting speed across the polar cap show maximum $10 \%$ variations.

\section{ACKNOWLEDGMENTS}

We thank the referee for detailed comments which improved the paper. DM acknowledges support and funding from the 'Indo-French Centre for the Promotion of Advanced Research - CEFIPRA' grant IFC/F5904-B/2018.

\section{REFERENCES}

Allen M. C., 1985, MNRAS, 215, 111

Arumugasamy P., Mitra D., 2019, MNRAS, 489, 4589

Asseo E., Melikidze G. I., 1998, MNRAS, 301, 59

Backer D. C., 1973, ApJ, 182, 245

Backer D. C., Rankin J. M., Campbell D. B., 1975, ApJ, 197, 481

Basu R., Mitra D., 2018, MNRAS, 475, 5098 

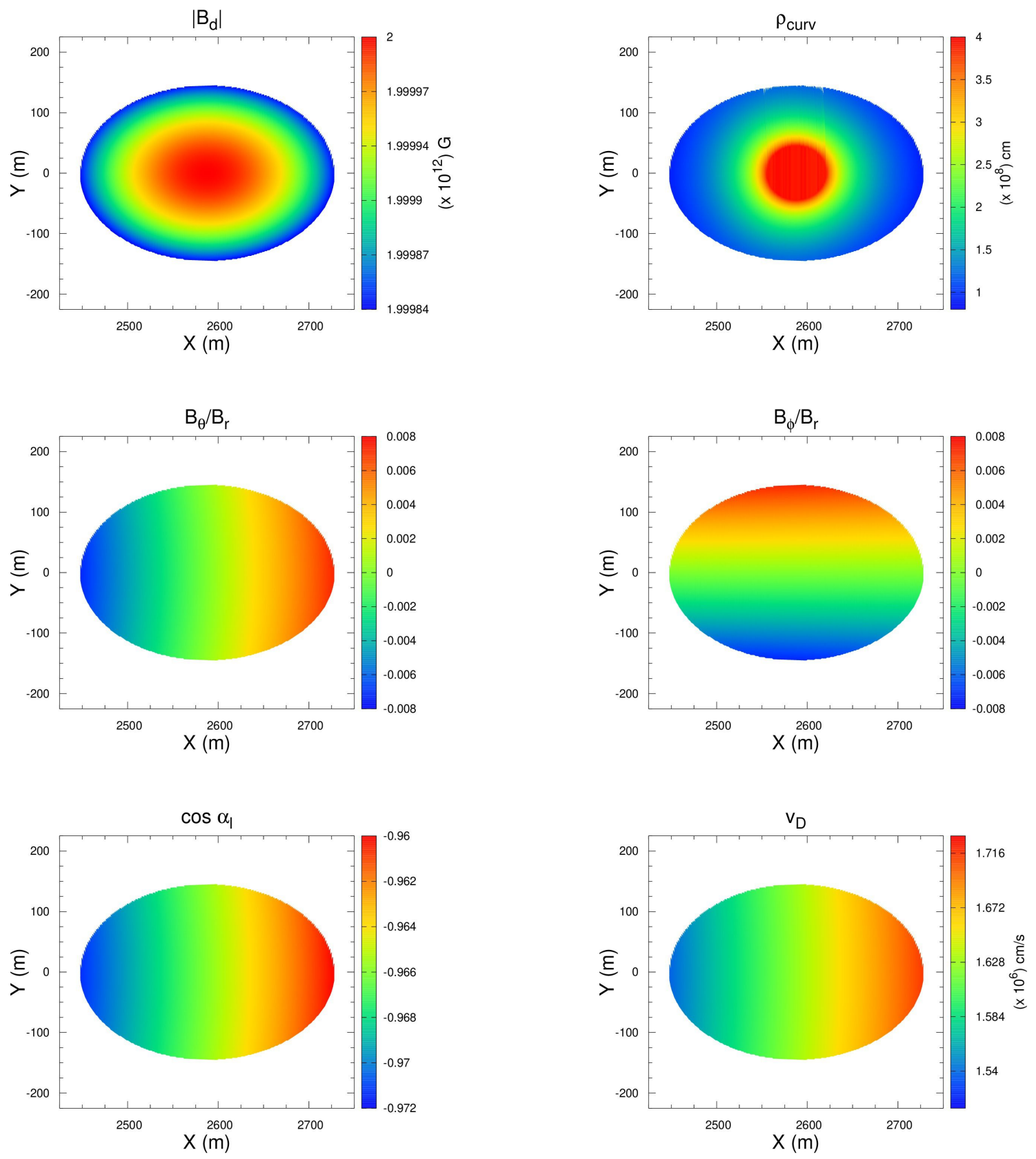

Figure 16. The figure shows the estimates of different physical parameters, magnetic field intensity ( $B_{d}$, top left), the radius of curvature of the field lines $\left(\rho_{\text {curv }}\right.$, top right), the ratio between the components of the magnetic field in spherical coordinates $\left(B_{\theta} / B_{r}\right.$, middle left and $B_{\phi} / B_{r}$, middle right), the inclination angle of surface magnetic field with the rotation axis ( $\cos \alpha_{l}$, bottom left) and the drift velocity of charges in presence of corotation electric field $\left(v_{D}\right.$, bottom right) in a dipolar polar cap. The magnetic moment has an inclination angle $\theta_{d}=15^{\circ}$ and all quantities are estimated at the surface of the neutron star, $R_{S}=10 \mathrm{~km}$, and the variations across the polar are represented by a colour scheme defined in each plot.

Basu R., Mitra D., Melikidze G. I., Maciesiak K., Skrzypczak A., Szary A., 2016, ApJ, 833, 29

Basu R., Mitra D., Melikidze G. I., Skrzypczak A., 2019a, MNRAS, 482, 3757

Basu R., Paul A., Mitra D., 2019b, MNRAS, 486, 5216

Champion D. J. et al., 2005, MNRAS, 363, 929

Cheng A. F., Ruderman M. A., 1977, ApJ, 214, 598

Cheng A. F., Ruderman M. A., 1980, ApJ, 235, 576

Drake F. D., Craft H. D., 1968, Nature, 220, 231
Gangadhara R. T., 2004, ApJ, 609, 335

Geppert U., 2017, Journal of Astrophysics and Astronomy, 38, 46 Gil J., Gronkowski P., Rudnicki W., 1984, A\&A, 132, 312

Gil J., Melikidze G. I., Geppert U., 2003, A\&A, 407, 315

Gil J. A., Melikidze G. I., Mitra D., 2002, A\&A, 388, 235

Gil J. A., Sendyk M., 2000, ApJ, 541, 351

Goldreich P., Julian W. H., 1969, ApJ, 157, 869

Kijak J., Gil J., 1997, MNRAS, 288, 631

Kijak J., Gil J., 2003, A\&A, 397, 969 

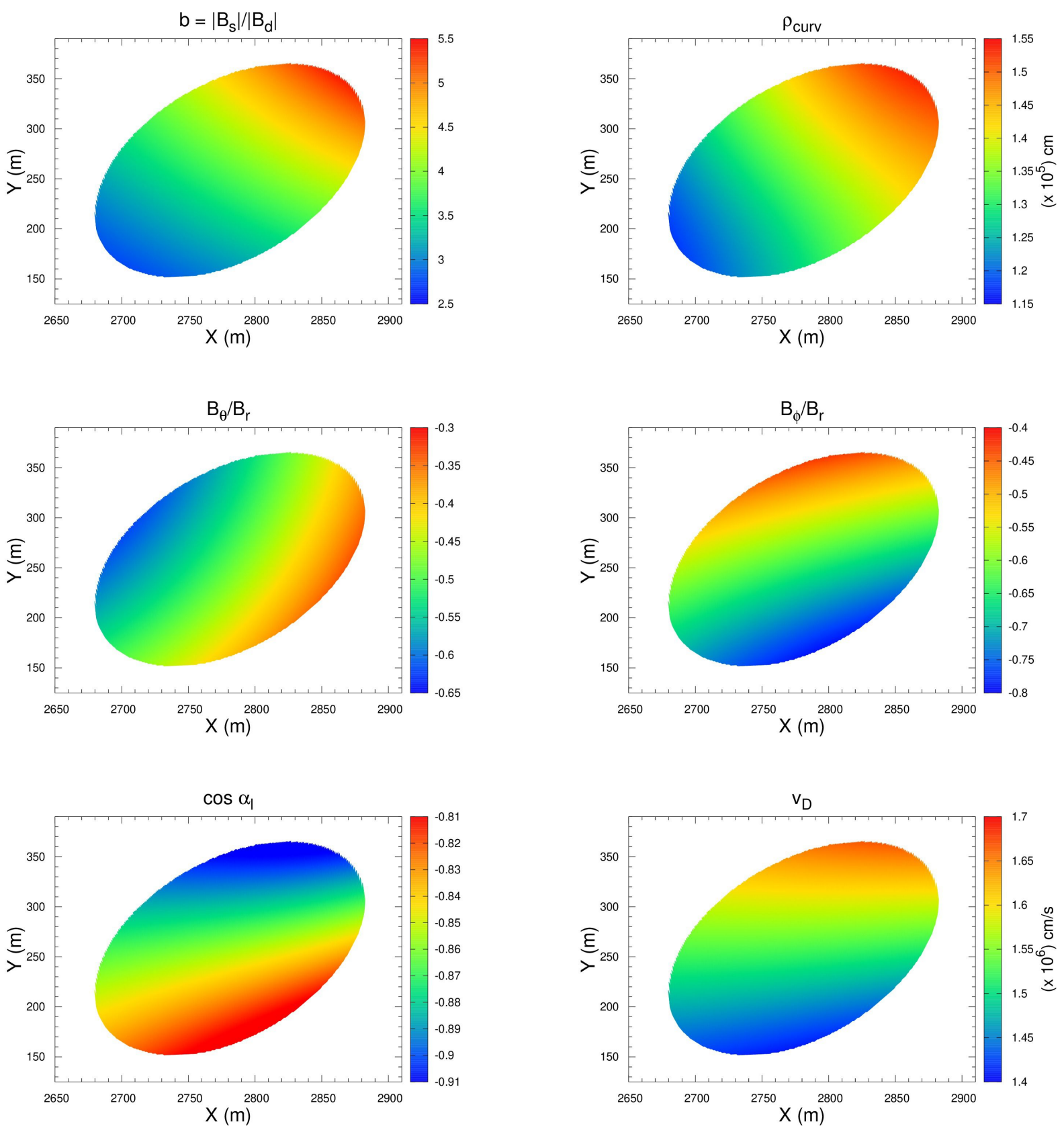

Figure 17. The figure shows the estimates of different physical parameters in a non-dipolar polar cap consisting of a combination of a star centered dipole with inclination angle $\theta_{d}=15^{\circ}$, and a dipole near the surface located at $\mathbf{r}_{s}=\left(0.95 R_{S}, 18.86^{\circ}, 10.99^{\circ}\right)$ with magnetic moment $\mathbf{m}_{s}=\left(0.001 d, 0^{\circ}, 0^{\circ}\right)$. The above magnetic field configuration results in coherent phase-modulated drifting. The different parameters are same as figure 16, with the only exception being the top right panel which shows ratio between non-dipolar magnetic field intensity and equivalent dipolar field $\left(\mathrm{b}=B_{s} / B_{d}\right)$.

Krzeszowski K., Mitra D., Gupta Y., Kijak J., Gil J., Acharyya A., 2009, MNRAS, 393, 1617

Lakoba T., Mitra D., Melikidze G., 2018, MNRAS, 480, 4526 Melikidze G. I., Gil J. A., Pataraya A. D., 2000, ApJ, 544, 1081 Melikidze G. I., Mitra D., Gil J., 2014, ApJ, 794, 105

Mitra D., 2017, Journal of Astrophysics and Astronomy, 38, 52 Mitra D., Basu R., Maciesiak K., Skrzypczak A., Melikidze G. I., Szary A., Krzeszowski K., 2016, ApJ, 833, 28

Mitra D., Basu R., Melikidze G. I., Arjunwadkar M., 2020, MNRAS, 492, 2468
Mitra D., Deshpande A. A., 1999, A\&A, 346, 906

Mitra D., Gil J., Melikidze G. I., 2009, ApJ, 696, L141

Mitra D., Li X. H., 2004, A\&A, 421, 215

Mitra D., Rankin J. M., 2002, ApJ, 577, 322

Pétri J., Mitra D., 2020, MNRAS, 491, 80

Rankin J. M., 1986, ApJ, 301, 901

Rankin J. M., 1993, ApJ, 405, 285

Ruderman M. A., Sutherland P. G., 1975, ApJ, 196, 51

Skrzypczak A., Basu R., Mitra D., Melikidze G. I., Maciesiak K., Koralewska O., Filothodoros A., 2018, ApJ, 854, 162 

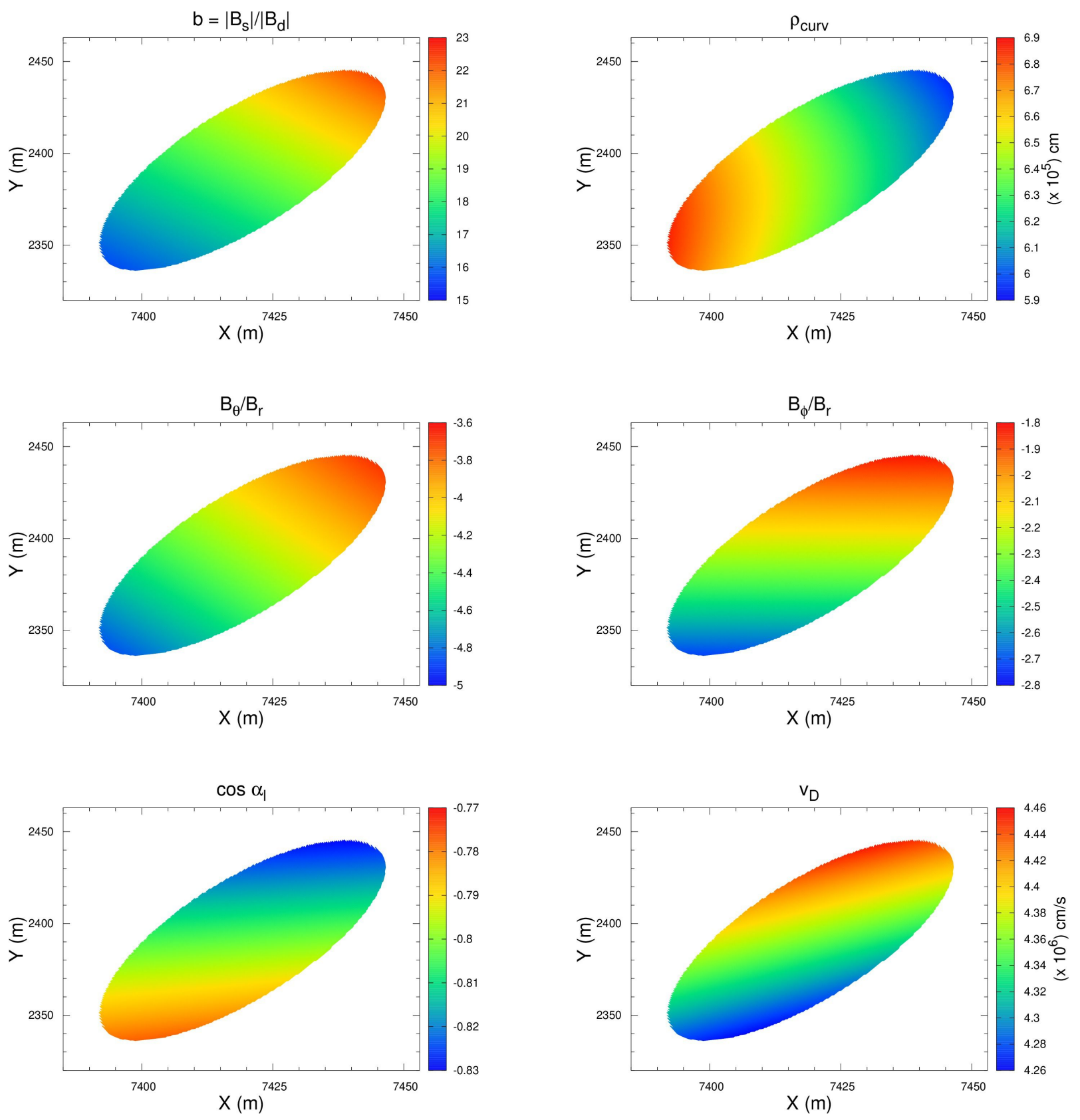

Figure 18. The figure shows the estimates of different physical parameters in a non-dipolar polar cap consisting of a combination of a star centered dipole with inclination angle $\theta_{d}=45^{\circ}$, and a dipole near the surface located at $\mathbf{r}_{s}=\left(0.95 R_{S}, 57.08^{\circ}, 20.66^{\circ}\right)$ with magnetic moment $\mathbf{m}_{s}=\left(0.05 d, 0^{\circ}, 0^{\circ}\right)$. The above magnetic field configuration results in low-mixed phase-modulated drifting. The different parameters are same as in figure 17 .

Sturrock P. A., 1971, ApJ, 164, 529

Szary A., 2013, Phd Thesis, Univ. of Zielona Góra, ArXiv e-prints

Szary A., van Leeuwen J., 2017, ApJ, 845, 95

Sznajder M., Geppert U., 2020, MNRAS, 493, 3770

Timokhin A. N., Harding A. K., 2019, ApJ, 871, 12

van Leeuwen J., Timokhin A. N., 2012, ApJ, 752, 155

Weltevrede P., 2016, A\&A, 590, A109

Weltevrede P., Edwards R. T., Stappers B. W., 2006, A\&A, 445, 243

Weltevrede P., Stappers B. W., Edwards R. T., 2007, A\&A, 469, 607 

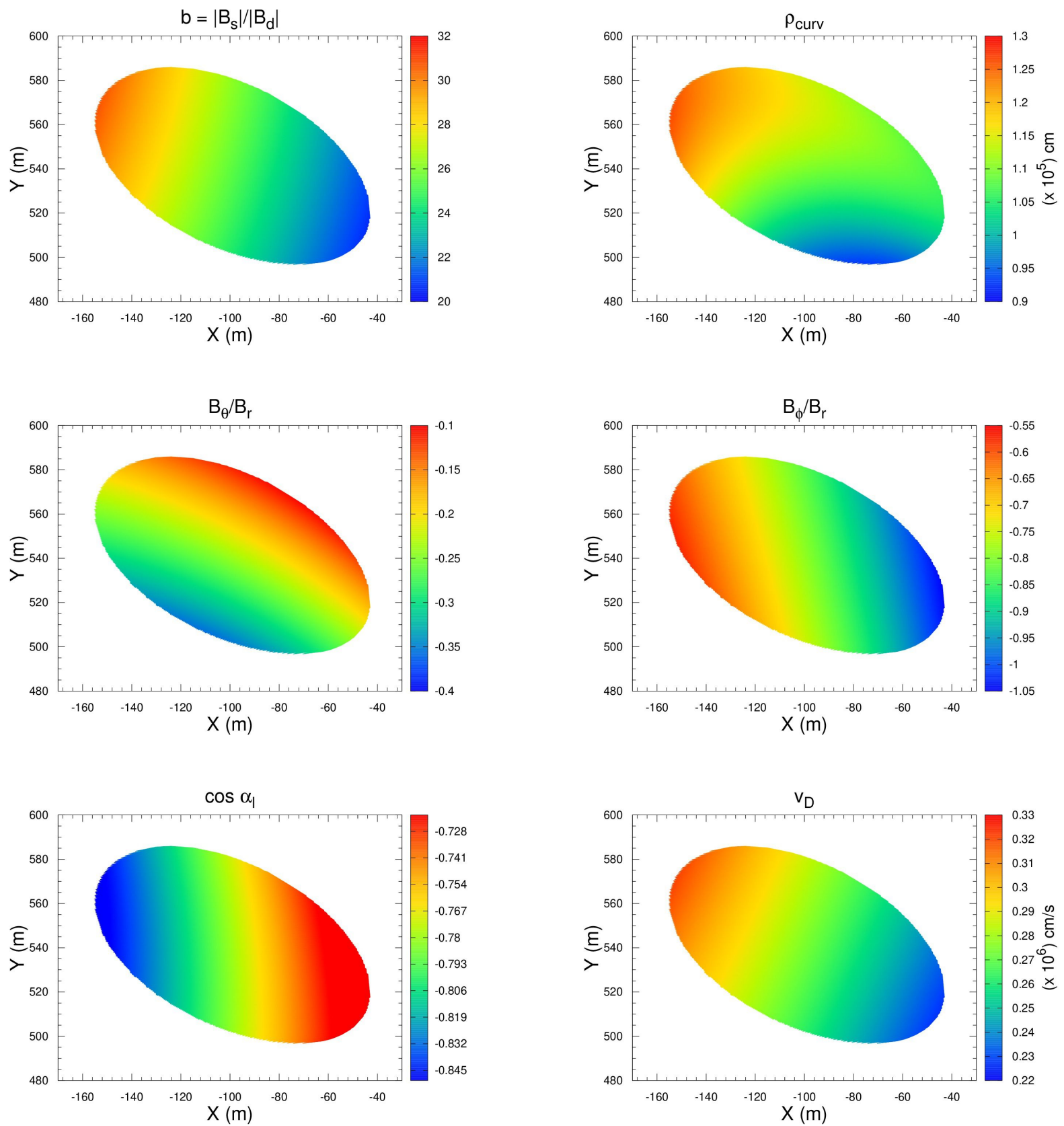

Figure 19. The figure shows the estimates of different physical parameters in a non-dipolar polar cap consisting of a combination of a star centered dipole with inclination angle $\theta_{d}=5^{\circ}$, and a dipole near the surface located at $\mathbf{r}_{s}=\left(0.95 R_{S}, 5^{\circ}, 120^{\circ}\right)$ with magnetic moment $\mathbf{m}_{s}=\left(0.005 \mathrm{~d}, 0^{\circ}, 0^{\circ}\right)$. The above magnetic field configuration results in switching phase-modulated drifting. The different parameters are same as in figure 17 and 18. 


\section{APPENDIX A: THE SURFACE MAGNETIC FIELD}

The basic estimates of the surface magnetic field configurations from a superposition of the star-centered global dipole and the crust-anchored dipole moment has been presented in Gil et al. (2002). The primary calculations were limited to the 2-dimensional representations. In this appendix we provide the more detailed 3-dimensional calculations. We also provide estimates for a general configuration of more than one crust-anchored dipole moments. The star centered global dipole with dipole moment $\mathbf{d}$ and magnetic inclination angle $\theta_{d}$, has the magnetic field at a point $\mathbf{r}=(r, \theta, \phi)$ given as :

$$
\begin{aligned}
B_{r}^{d} & =\frac{2 d}{r^{3}}\left(\sin \theta_{d} \sin \theta \cos \phi+\cos \theta_{d} \cos \theta\right) \\
B_{\theta}^{d} & =-\frac{d}{r^{3}}\left(\sin \theta_{d} \cos \theta \cos \phi-\cos \theta_{d} \sin \theta\right) \\
B_{\phi}^{d} & =\frac{d}{r^{3}} \sin \theta_{d} \sin \phi
\end{aligned}
$$

In a more generalised situation the surface fields are nondipolar in nature and can be approximated by one or more crust-anchored dipoles. Assuming the possibility of a series of $N$ dipoles with dipole moment $\mathbf{m}_{i}=\left(m^{i}, \theta_{m}^{i}, \phi_{m}^{i}\right)$ at location $\mathbf{r}_{i}=\left(r^{i}, \theta_{r}^{i}, \phi_{r}^{i}\right)$ where $i=1,2, \ldots, N$; the components of the radius-vector and the local dipole moment for the $i^{\text {th }}$ dipole can be expressed as :

$$
\begin{aligned}
r_{r}^{i} & =r^{i}\left(\sin \theta_{r}^{i} \sin \theta \cos \left(\phi-\phi_{r}^{i}\right)+\cos \theta_{r}^{i} \cos \theta\right), \\
r_{\theta}^{i} & =r^{i}\left(\sin \theta_{r}^{i} \cos \theta \cos \left(\phi-\phi_{r}^{i}\right)-\cos \theta_{r}^{i} \sin \theta\right), \\
r_{\phi}^{i} & =-r^{i} \sin \theta_{r}^{i} \sin \left(\phi-\phi_{r}^{i}\right) . \\
m_{r}^{i} & =m^{i}\left(\sin \theta_{m}^{i} \sin \theta \cos \left(\phi-\phi_{m}^{i}\right)+\cos \theta_{m}^{i} \cos \theta\right), \\
m_{\theta}^{i} & =m^{i}\left(\sin \theta_{m}^{i} \cos \theta \cos \left(\phi-\phi_{m}^{i}\right)-\cos \theta_{m}^{i} \sin \theta\right), \\
m_{\phi}^{i} & =-m^{i} \sin \theta_{m}^{i} \sin \left(\phi-\phi_{m}^{i}\right) .
\end{aligned}
$$

The above expressions are used to define

$$
\begin{aligned}
D_{i} & =\left(r^{i}\right)^{2}+r^{2}-2 r^{i} r\left(\sin \theta_{r}^{i} \sin \theta \cos \left(\phi-\phi_{r}^{i}\right)+\cos \theta_{r}^{i} \cos \theta\right), \\
T_{i} & =m_{r}^{i} r-\left(m_{r}^{i} r_{r}^{i}+m_{\theta}^{i} r_{\theta}^{i}+m_{\phi}^{i} r_{\phi}^{i}\right) .
\end{aligned}
$$

The magnetic field $\mathbf{B}_{i}=\left(B_{r}^{i}, B_{\theta}^{i}, B_{\phi}^{i}\right)$ for the $i^{\text {th }}$ dipole can be characterised as follows:

$$
\begin{aligned}
& B_{r}^{i}=-\frac{1}{D_{i}^{2.5}}\left(3 T_{i} r_{r}^{i}-3 T_{i} r+D_{i} m_{r}^{i}\right), \\
& B_{\theta}^{i}=-\frac{1}{D_{i}^{2.5}}\left(3 T_{i} r_{\theta}^{i}+D_{i} m_{\theta}^{i}\right), \\
& B_{\phi}^{i}=-\frac{1}{D_{i}^{2.5}}\left(3 T_{i} r_{\phi}^{i}+D_{i} m_{\phi}^{i}\right) .
\end{aligned}
$$

The magnetic field can be used to determine the field line of force which are obtained in spherical coordinate system by solving the differential equations :

$$
\begin{aligned}
& \frac{\mathrm{d} \theta}{\mathrm{d} r}=\frac{B_{\theta}^{d}+\sum_{i=1}^{N} B_{\theta}^{i}}{r\left(B_{r}^{d}+\sum_{i=1}^{N} B_{r}^{i}\right)} \equiv \Theta_{1}, \\
& \frac{\mathrm{d} \phi}{\mathrm{d} r}=\frac{B_{\phi}^{d}+\sum_{i=1}^{N} B_{\phi}^{i}}{r\left(B_{r}^{d}+\sum_{i=1}^{N} B_{r}^{i}\right) \sin \theta} \equiv \Phi_{1} .
\end{aligned}
$$

\section{APPENDIX B: CURVATURE IN MAGNETIC FIELD}

Finally, we present a detailed calculation of the curvature of the magnetic field lines which is important for the pair production process in the IAR (see RS75). Following the specifications of Gil et al. (2002) the curvature $\Re\left(=1 / \rho_{c}\right.$, $\rho_{c}$ being the radius of curvature) is given as :

$\Re=\left(\frac{\mathrm{d} s}{\mathrm{~d} r}\right)^{-3}\left|\left(\frac{\mathrm{d}^{2} \mathbf{r}}{\mathrm{d} r^{2}} \frac{\mathrm{d} s}{\mathrm{~d} r}-\frac{\mathrm{d} \mathbf{r}}{\mathrm{d} r} \frac{\mathrm{d}^{2} s}{\mathrm{~d} r^{2}}\right)\right|$

which gives $\Re=\left(S_{1}\right)^{-3}\left(J_{1}^{2}+J_{2}^{2}+J_{3}^{2}\right)^{1 / 2}$. The expressions are specified as $J_{1}=X_{2} S_{1}-X_{1} S_{2} ; J_{2}=Y_{2} S_{1}-Y_{1} S_{2}$ and $J_{3}=Z_{2} S_{1}-Z_{1} S_{2}$, where

$$
\begin{aligned}
X_{1}= & \sin \theta \cos \phi+r \Theta_{1} \cos \theta \cos \phi-r \Phi_{1} \sin \theta \sin \phi, \\
Y_{1}= & \sin \theta \sin \phi+r \Theta_{1} \cos \theta \sin \phi+r \Phi_{1} \sin \theta \cos \phi, \\
Z_{1}= & \cos \theta-r \Theta_{1} \sin \theta, \\
X_{2}= & \left(2 \Theta_{1}+r \Theta_{2}\right) \cos \theta \cos \phi-\left(2 \Phi_{1}+r \Phi_{2}\right) \sin \theta \sin \phi \\
& -r\left(\Theta_{1}^{2}+\Phi_{1}^{2}\right) \sin \theta \cos \phi-2 r \Theta_{1} \Phi_{1} \cos \theta \sin \phi, \\
Y_{2}= & \left(2 \Theta_{1}+r \Theta_{2}\right) \cos \theta \sin \phi+\left(2 \Phi_{1}+r \Phi_{2}\right) \sin \theta \cos \phi \\
& -r\left(\Theta_{1}^{2}+\Phi_{1}^{2}\right) \sin \theta \sin \phi+2 r \Theta_{1} \Phi_{1} \cos \theta \cos \phi, \\
Z_{2}= & -2 \Theta_{1} \sin \theta-r \Theta_{2} \sin \theta-r \Theta_{1}^{2} \cos \theta .
\end{aligned}
$$

Here $\Theta_{1}=\mathrm{d} \theta / \mathrm{d} r$ and $\Phi_{1}=\mathrm{d} \phi / \mathrm{d} r$ are specified in Eq.(A5). Additionally, $S_{1}=\mathrm{d} s / \mathrm{d} r$ and $S_{2}=\mathrm{d}^{2} s / \mathrm{d} r^{2}$ are given as :

$$
\begin{aligned}
S_{1}= & \left(1+r^{2} \Theta_{1}^{2}+r^{2} \Phi_{1}^{2} \sin ^{2} \theta\right)^{1 / 2}, \\
S_{2}=S_{1}^{-1}\left(r \Theta_{1}^{2}+r^{2} \Theta_{1} \Theta_{2}+r \Phi_{1}^{2} \sin ^{2} \theta\right. & \\
& \left.\quad+r^{2} \Phi_{1} \Phi_{2} \sin ^{2} \theta+r^{2} \Theta_{1} \Phi_{1}^{2} \sin \theta \cos \theta\right) .
\end{aligned}
$$

Finally, we have estimated $\Theta_{2}=\mathrm{d} \Theta_{1} / \mathrm{d} r$ and $\Phi_{2}=\mathrm{d} \Phi_{1} / \mathrm{d} r$ as follows :

$$
\begin{gathered}
\Theta_{2}=-\frac{\Theta_{1}}{r}+\frac{1}{r\left(B_{r}^{d}+\sum_{i=1}^{N} B_{r}^{i}\right)}\left(\frac{\mathrm{d} B_{\theta}^{d}}{\mathrm{~d} r}+\sum_{i=1}^{N} \frac{\mathrm{d} B_{\theta}^{i}}{\mathrm{~d} r}\right) \\
-\frac{B_{\theta}^{d}+\sum_{i=1}^{N} B_{\theta}^{i}}{r\left(B_{r}^{d}+\sum_{i=1}^{N} B_{r}^{i}\right)^{2}}\left(\frac{\mathrm{d} B_{r}^{d}}{\mathrm{~d} r}+\sum_{i=1}^{N} \frac{\mathrm{d} B_{r}^{i}}{\mathrm{~d} r}\right), \\
\Phi_{2}=-\frac{\Phi_{1}}{r}+\frac{1}{r\left(B_{r}^{d}+\sum_{i=1}^{N} B_{r}^{i}\right)}\left(\frac{\mathrm{d} B_{\phi}^{d}}{\mathrm{~d} r}+\sum_{i=1}^{N} \frac{\mathrm{d} B_{\phi}^{i}}{\mathrm{~d} r}\right) \\
-\frac{B_{\phi}^{d}+\sum_{i=1}^{N} B_{\phi}^{i}}{r\left(B_{r}^{d}+\sum_{i=1}^{N} B_{r}^{i}\right)^{2}}\left(\frac{\mathrm{d} B_{r}^{d}}{\mathrm{~d} r}+\sum_{i=1}^{N} \frac{\mathrm{d} B_{r}^{i}}{\mathrm{~d} r}\right) .
\end{gathered}
$$

where,

$$
\begin{aligned}
\frac{\mathrm{d} B_{r}^{d}}{\mathrm{~d} r} & =-\frac{3 B_{r}^{d}}{r}-2 B_{\theta}^{d} \Theta_{1}-2 B_{\phi}^{d} \cos \theta \Phi_{1}, \\
\frac{\mathrm{d} B_{\theta}^{d}}{\mathrm{~d} r} & =\frac{3 B_{\theta}^{d}}{r}+\frac{1}{2} B_{r}^{d} \Theta_{1}+B_{\phi}^{d} \cos \theta \Phi_{1}, \\
\frac{\mathrm{d} B_{\phi}^{d}}{\mathrm{~d} r} & =-\frac{3 B_{\phi}^{d}}{r}+\frac{d}{r^{3}} \sin \theta_{d} \cos \phi \Phi_{1} .
\end{aligned}
$$


and

$$
\begin{aligned}
\frac{\mathrm{d} B_{r}^{i}}{\mathrm{~d} r}= & -\frac{2.5}{D_{i}} B_{r}^{i} \frac{\mathrm{d} D_{i}}{\mathrm{~d} r}-\frac{1}{D_{i}^{2.5}}\left(3 \frac{\mathrm{d} T_{i}}{\mathrm{~d} r} r_{r}^{i}+3 T_{i} \frac{\mathrm{d} r_{i}^{r}}{\mathrm{~d} r}\right. \\
& \left.-3 \frac{\mathrm{d} T_{i}}{\mathrm{~d} r} r-3 T_{i}+\frac{\mathrm{d} D_{i}}{\mathrm{~d} r} m_{r}^{i}+D_{i} \frac{\mathrm{d} m_{r}^{i}}{\mathrm{~d} r}\right), \\
\frac{\mathrm{d} B_{\theta}^{i}}{\mathrm{~d} r}= & -\frac{2.5}{D_{i}} B_{\theta}^{i} \frac{\mathrm{d} D_{i}}{\mathrm{~d} r}-\frac{1}{D_{i}^{2.5}}\left(3 \frac{\mathrm{d} T_{i}}{\mathrm{~d} r} r_{\theta}^{i}+3 T_{i} \frac{\mathrm{d} r_{i}^{\theta}}{\mathrm{d} r}\right. \\
& \left.+\frac{\mathrm{d} D_{i}}{\mathrm{~d} r} m_{\theta}^{i}+D_{i} \frac{\mathrm{d} m_{\theta}^{i}}{\mathrm{~d} r}\right), \\
\frac{\mathrm{d} B_{\phi}^{i}}{\mathrm{~d} r}= & -\frac{2.5}{D_{i}} B_{\phi}^{i} \frac{\mathrm{d} D_{i}}{\mathrm{~d} r}-\frac{1}{D_{i}^{2.5}}\left(3 \frac{\mathrm{d} T_{i}}{\mathrm{~d} r} r_{\phi}^{i}+3 T_{i} \frac{\mathrm{d} r_{i}^{\phi}}{\mathrm{d} r}\right. \\
& \left.+\frac{\mathrm{d} D_{i}}{\mathrm{~d} r} m_{\phi}^{i}+D_{i} \frac{\mathrm{d} m_{\phi}^{i}}{\mathrm{~d} r}\right) . \\
\frac{\mathrm{d} D_{i}}{\mathrm{~d} r}= & 2 r-2 r_{r}^{i}-2 r \frac{\mathrm{d} r_{r}^{i}}{\mathrm{~d} r}, \\
\frac{\mathrm{d} T_{i}}{\mathrm{~d} r}= & m_{r}^{i}+r \frac{\mathrm{d} m_{r}^{i}}{\mathrm{~d} r}-\left(\frac{\mathrm{d} m_{r}^{i}}{\mathrm{~d} r} r_{r}^{i}+m_{r}^{i} \frac{\mathrm{d} r_{r}^{i}}{\mathrm{~d} r}\right. \\
& \left.+\frac{\mathrm{d} m_{\theta}^{i}}{\mathrm{~d} r} r_{\theta}^{i}+m_{\theta}^{i} \frac{\mathrm{d} r_{\theta}^{i}}{\mathrm{~d} r}+\frac{\mathrm{d} m_{\phi}^{i}}{\mathrm{~d} r} r_{\phi}^{i}+m_{\phi}^{i} \frac{\mathrm{d} r_{\phi}^{i}}{\mathrm{~d} r}\right) .
\end{aligned}
$$

$\frac{\mathrm{d} r_{r}^{i}}{\mathrm{~d} r}=r_{\theta}^{i} \Theta_{1}+r_{\phi}^{i} \sin \theta \Phi_{1}$,

$\frac{\mathrm{d} r_{\theta}^{i}}{\mathrm{~d} r}=-r_{r}^{i} \Theta_{1}+r_{\phi}^{i} \cos \theta \Phi_{1}$,

$\frac{\mathrm{d} r_{\phi}^{i}}{\mathrm{~d} r}=-r^{i} \sin \theta_{r}^{i} \cos \left(\phi-\phi_{r}^{i}\right) \Phi_{1}$.

$\frac{\mathrm{d} m_{r}^{i}}{\mathrm{~d} r}=m_{\theta}^{i} \Theta_{1}+m_{\phi}^{i} \sin \theta \Phi_{1}$,

$\frac{\mathrm{d} m_{\theta}^{i}}{\mathrm{~d} r}=-m_{r}^{i} \Theta_{1}+m_{\phi}^{i} \cos \theta \Phi_{1}$,

$\frac{\mathrm{d} m_{\phi}^{i}}{\mathrm{~d} r}=-m^{i} \sin \theta_{m}^{i} \cos \left(\phi-\phi_{m}^{i}\right) \Phi_{1}$. 